\title{
Hadronic Multiparticle Production at Ultra-High Energies and Extensive Air Showers
}

\author{
Ralf Ulrich ${ }^{\mathrm{a}}$, Ralph Engel ${ }^{\mathrm{b}}$, and Michael Unger $^{\mathrm{b}}$ \\ a The Pennsylvania State University, Center for Particle Astrophysics, \\ 104 Davey Lab, University Park, PA 16802, USA and \\ b Karlsruhe Institute of Technology (KIT), Institut für Kernphysik, P.O. Box 3640, 76021 Karlsruhe, Germany
}

(Dated: November 7, 2018)

\begin{abstract}
Studies of the nature of cosmic ray particles at the highest energies are based on the measurement of extensive air showers. Most cosmic ray properties can therefore only be obtained from the interpretation of air shower data and are thus depending on predictions of hadronic interaction models at ultra-high energies. We discuss different scenarios of model extrapolations from accelerator data to air shower energies and investigate their impact on the corresponding air shower predictions. To explore the effect of different extrapolations by hadronic interaction models we developed an ad hoc model. This ad hoc model is based on the modification of the output of standard hadronic interaction event generators within the air shower simulation process and allows us to study the impact of changing interaction features on the air shower development. In a systematic study we demonstrate the resulting changes of important air shower observables and also discuss them in terms of the predictions of the Heitler model of air shower cascades. It is found that the results of our ad hoc modifications are, to a large extend, independent of the choice of the underlying hadronic interaction model.
\end{abstract}

\section{INTRODUCTION}

The nature of the highest energy cosmic ray particles is still an open question. Even with recent high exposure experiments delivering large quantities of data at ultra-high energies [1] 3], the fundamental problem remains to interpret the collected air shower data. This is mainly due to difficulties and limitations in modelling of hadronic interactions in air shower cascades. Whereas we can predict reliably electromagnetic interactions with QED, it is still not possible to calculate hadronic multiparticle production from first principles. Moreover, most of the interaction energies and phase space regions of relevance to the development of air showers are not directly accessible in accelerator experiments. Thus, phenomenological models of hadronic interactions have to be used to extrapolate the available accelerator measurements to these unexplored phase space regions.

Due to the lack of a theoretical framework that allows one to make quantitative predictions, the systematic uncertainties of these extrapolations are very difficult to estimate. This is a well known problem for, e.g. the determination of the systematic uncertainty of the primary mass composition derived from air shower measurements [ 3 [ 9 . Similarly, the reconstruction of the energy of the primary particle from detector data calibrated by Monte Carlo simulations is subject to largely unknown model uncertainties 9 -14].

For estimating systematic uncertainties of air shower predictions, one first needs to know the uncertainties of the model predictions for multiparticle production, and secondly how these uncertainties propagate to predictions for different air shower observables. In this work we will address the latter by studying the relation of different extrapolations of hadronic particle production to air shower predictions.

We will briefly discuss the uncertainties associated with the modelling of hadronic interactions and argue that the differences of existing models do not cover the full range of the expected uncertainty at energies beyond the reach of colliders. To nevertheless explore a wide range of different extrapolations and to calculate the corresponding air shower observables, we will introduce an ad hoc modification of existing models. The shower observables under consideration are the depth of shower maximum and the number of muons at ground level-as the model-dependence of the interpretation of air shower data is mainly related to these two parameters [15]. All other characteristics of air showers are closely related to those two features due to universality of the electromagnetic shower component (see [16 21] and Refs. therein). The results will also be compared to the expectations from the Heitler-Matthews cascade model [22].

\section{UNCERTAINTIES IN MODELLING HADRONIC INTERACTIONS}

The development of extensive air showers (EAS) is very sensitive to the characteristics of hadronic interactions at ultra-high energies. Different hadronic interaction event generators are available for the simulation of EAS.

Currently it is unclear whether the existing differences in model predictions for hadronic multiparticle production can be used to estimate the level of theoretical uncertainties related to our limited understanding of hadronic interactions in air showers. It is possible that existing model-differences are

(A) larger than the actual systematic uncertainties. For example, progress in understanding hadronic particle production and new data from accelerators allow us to update interaction models to obtain a more realistic description of particle production. 

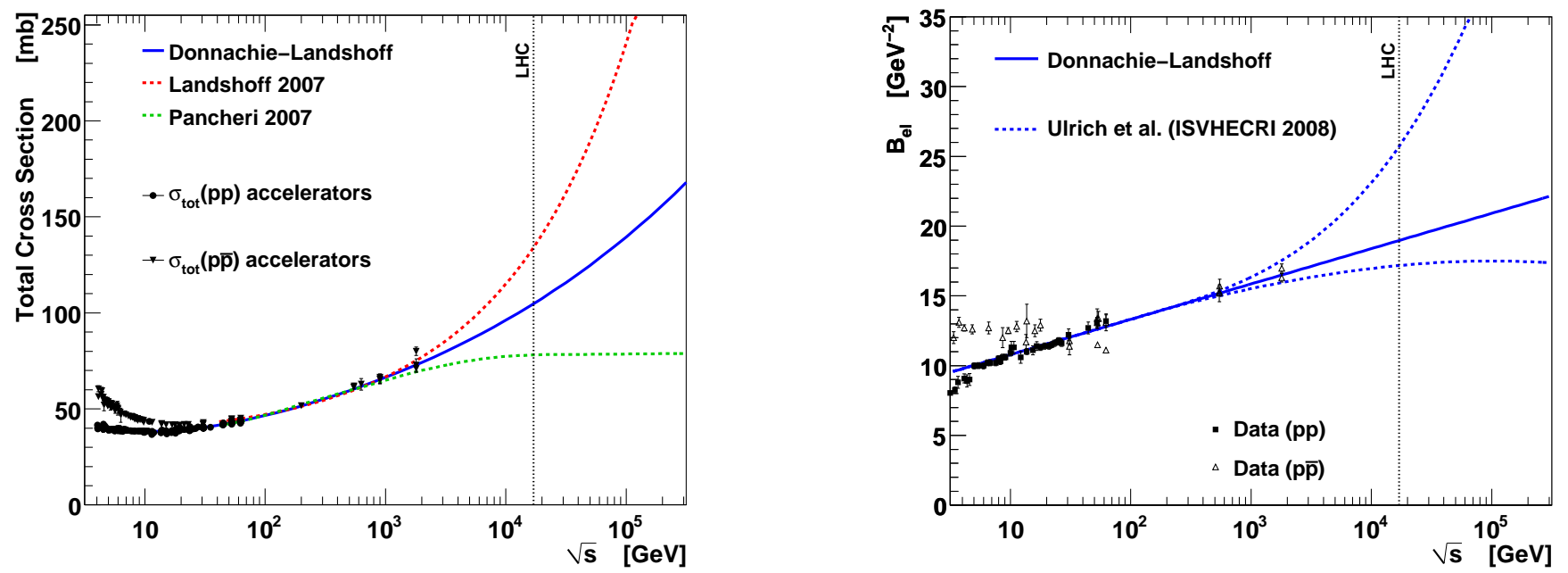

FIG. 1. Compilation of accelerator data of $\sigma_{\text {tot }}^{\mathrm{pp}}$ and $B_{\mathrm{el}}[23]$. The central line denotes the conventional extrapolation of these data to high energy. The upper and lower lines indicate a set of possible extreme extrapolations. In the left plot the conventional model is the soft pomeron parametrization by Donnachie and Landshoff [24], while the lower curve is by Pancheri et al. 25] and the upper one is the two-pomeron model of Landshoff [26, 27]. The different scenarios in the right plot are from [28].

Not all models are updated regularly and the quality of data description differs between the models.

(B) smaller than the actual systematic uncertainties. The existing models are not covering the full phase space of possible interaction scenarios and parameters. Moreover, new physics processes at higher energies, which are unknown now and thus missing in current modelling approaches, could change extrapolations drastically.

Frequently used models for the high-energy range are QGSJet II [29, 30], Epos [31], and the somewhat older QGSJet 01 [32, 33] and Sibyll 2.1 [34]. These models are available in the air shower simulation package CONEX [35] that will be used for calculating the shower observables. Other models for hadronic interactions include NEXUs [36, 37], HDPM [38], DPMJET [39], and VENUS [40]. These models are older or more limited in the scope of application and not considered here.

Despite the different level of sophistication, the predictions by Sibyll 2.1 and QGSJET 01 are not objectively worse than those by QGSJETII and Epos, as many model aspects are assumptions that cannot be justified by underlying fundamental theoretical constraints. Over the years model predictions and extrapolations have become more alike even though there is no theory for calculating e.g. cross sections from first principles [41]. One has to be careful and should not consider this increasing similarity of model predictions as real convergence and significant decrease of the uncertainties. None of the models is able to consistently describe cosmic ray data (e.g. 7, 9, 42, 43]). In the energy range up to about $10^{15} \mathrm{eV}$ where various measurements on multipar- ticle production are available good tuning to many different data sets should indeed lead to a convergence of the model predictions. However, at energies beyond that of collider experiments, the extrapolations can only be guided by theoretical end phenomenological assumptions.

In Fig. 1 accelerator data on the total proton-proton cross section $\sigma_{\mathrm{tot}}^{\mathrm{pp}}$ and the elastic slope parameter $B_{\mathrm{el}}$, defined by $\mathrm{d} \sigma_{\mathrm{el}} /\left.\mathrm{d} t\right|_{t=0} \propto \exp \left(-|t| B_{\text {el }}\right)$, are shown together with different models that extrapolate these data to ultra-high energies. Converting these model extrapolations within the Glauber framework [44, 45] to proton-

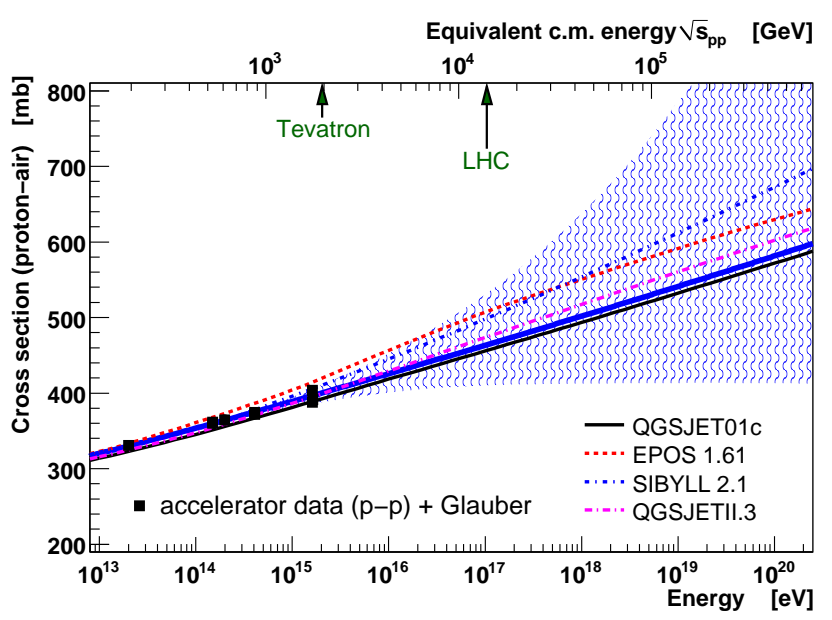

FIG. 2. Uncertainty of the extrapolation of the proton-air cross section for particle production due to different models of the proton-proton cross section as calculated with the Glauber framework [28]. 
air cross sections for particle production as needed for air shower simulation leads to a wide range of predictions at ultra-high energy [28]. This range is shown in Fig. 2] as the shaded area. The extrapolations of aforementioned interaction models are also shown and it is obvious that they do not exhaust the possible range of uncertainties. In addition it can be seen that a measurement of the total cross section at LHC energy with an uncertainty smaller than a few percent has the potential to significantly reduce the uncertainties of the model extrapolations at cosmic ray energies. Furthermore, recent activities of model development of the EPos event generator clearly demonstrated that there exists a large freedom to change predictions at ultra-high energies [46, 47].

All this is demonstrating that the current situation is most likely such that one would underestimate the systematic uncertainties of model extrapolations at energies beyond the reach of accelerator experiments if one would just consider the extrapolation of existing event generators. This is supported by the fact that existing interaction models are not able to consistently describe cosmic ray observations. Therefore it is also not surprising that first LHC data [48 55] indicate some model deficiencies [56].

In the following we will consider different extrapolations of multiparticle production to energies higher than $10^{15} \mathrm{eV}$, corresponding to a c.m.s. energy of about $2 \mathrm{TeV}$ for proton-proton collisions. We will concentrate on general features of hadronic particle production that are most directly linked to air shower predictions.

\section{PREDICTIONS OF THE CASCADE MODEL FOR AIR SHOWERS}

Simple cascade models, often referred to as Heitler models [57], are providing some insight into how air shower observables are related to interaction physics on a microscopic level [22, 58, 59]. The descriptive strength of Heitler models-despite their extreme simplicity-is remarkable. We will discuss the ability of such models to link the physics of interactions to air shower observables, but also point out the limitations of these models.

\section{A. Electromagnetic Heitler Model}

In the electromagnetic Heitler approximation only one particle type is considered and substitutes $\gamma, \mathrm{e}^{+}$and $\mathrm{e}^{-}$. It is assumed that an e.m. particle with energy $E$ interacts after one splitting length $\lambda_{\mathrm{e}}=\ln 2 X_{0}$, where $X_{0} \sim 37 \mathrm{~g} / \mathrm{cm}^{2}$ is the e.m. radiation length, producing two secondaries with energy $E / 2$, see Fig. 3 The number of particles after each splitting length increases by a factor of two, and thus the number of particles at generation $n$ is

$$
N_{n}=2^{n}
$$

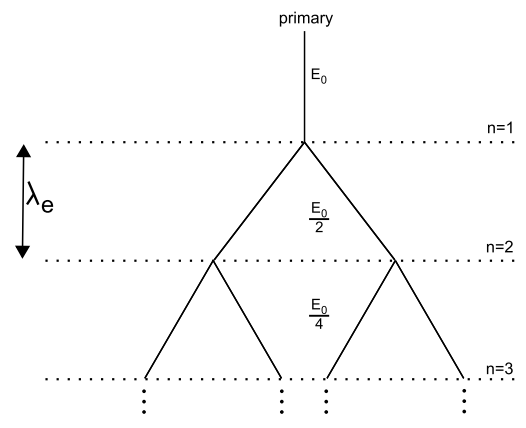

FIG. 3. Electromagnetic Heitler model.

and their energy is

$$
E_{n}=E_{0} / N_{n} .
$$

Defining the critical energy $E_{\mathrm{c}}^{\text {e.m. }}$ as the energy below which energy loss processes dominate over particle production ( $\sim 85 \mathrm{MeV}$ in air), one can make the assumption that the shower maximum is reached when the energy of secondary particles reaches $E_{\mathrm{c}}^{\text {e.m. }}$. Thus, two main shower observables are given by

$$
\begin{aligned}
& N_{\max }=E_{0} / E_{\mathrm{c}}^{\text {e.m. }} \sim E_{0} \quad \text { and } \\
& X_{\max }=\lambda_{\mathrm{e}} n_{\mathrm{c}} \sim \lambda_{\mathrm{e}} \ln \left(E_{0}\right),
\end{aligned}
$$

with $n_{\mathrm{c}}=(\ln 2)^{-1} \ln \left(E_{0} / E_{\mathrm{c}}^{\text {e.m. }}\right)$. Even this very simplistic model reproduces two important features of air showers: the number of particles at shower maximum $N_{\max }$ is proportional to $E_{0}$ and the depth of shower maximum $X_{\max }$ depends logarithmically on the primary energy $E_{0}$.

\section{B. Hadronic Extension of the Heitler Model}

The Heitler model can be extended to hadronic particles by considering a cascade of pions interacting in air 22]. It is assumed that a charged pion $\left(\pi^{+}\right.$or $\pi^{-}$) of energy $E$ interacts after one interaction length $\lambda_{\mathrm{I}}\left(\approx 120 \mathrm{~g} / \mathrm{cm}^{2}\right.$ for pions of $\left.10-1000 \mathrm{GeV}[60]\right)$ and produces $r n_{\text {mult }}$ charged pions and $c n_{\text {mult }}$ neutral pions, see Fig. 4. Here $c=1-r$ is the pion charge-ratio and is in the Heitler framework typically defined to be $1 / 3$. Neutral pions decay instantaneously into $2 \gamma$ and are thus lost from the hadronic cascade, while transferring energy into the electromagnetic cascade. The number of charged pions in the hadronic cascade at generation $n$ is

$$
N_{n}^{\mathrm{ch}}=\left(r n_{\text {mult }}\right)^{n}
$$

and the energy per pion

$$
E_{n}^{\pi}=\frac{E_{0}}{\left(n_{\text {mult }}\right)^{n}} .
$$

It is assumed that charged pions decay into muons as soon as their energy drops below $E_{\mathrm{dec}}$. This is the 


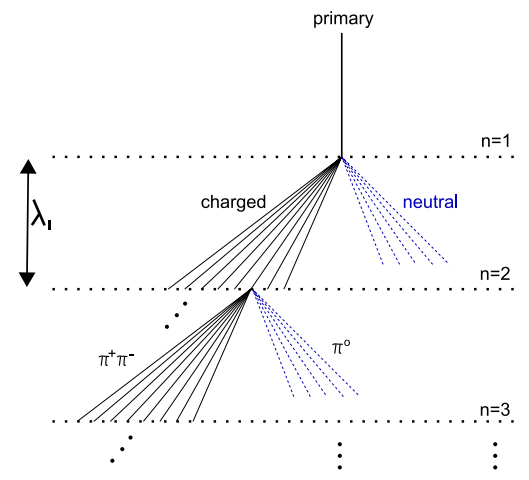

FIG. 4. Hadronic Heitler-Matthews model: pion cascade in air.

characteristic energy where the interaction length becomes larger than the decay length of pions in air, and is thus also depending on the atmospheric density profile. For example high up in the atmosphere at a depth of $\sim 100 \mathrm{~g} / \mathrm{cm}^{2}$ this energy is around $E_{\mathrm{dec}} \sim 55 \mathrm{GeV}$ and decreases to $E_{\mathrm{dec}} \sim 18 \mathrm{GeV}$ at sea level. In realistic air shower scenarios the energy $E_{n}^{\pi}=E_{\mathrm{dec}}$ is typically reached within the range of $20-30 \mathrm{GeV}$ [22, 60, 61], which is then adopted to be the critical energy of pions $E_{\mathrm{c}}^{\pi}$, in analogy to the critical energy of electrons $E_{\mathrm{c}}^{\mathrm{e} . \mathrm{m}}$. The number of generations required to reach $E_{n}^{\pi}=E_{\mathrm{c}}^{\pi}$ is

$$
n_{\mathrm{c}}=\frac{\ln \left(E_{0} / E_{\mathrm{c}}^{\pi}\right)}{\ln n_{\mathrm{mult}}}
$$

and is for air showers in the range of 4 to 7 [62] This yields a muon number from decaying charged pions of

$$
\begin{aligned}
N_{\mu} & =N_{n_{\mathrm{c}}}^{\mathrm{ch}}=\left(r n_{\text {mult }}\right)^{n_{\mathrm{c}}}=\left(\frac{E_{0}}{E_{\mathrm{c}}^{\pi}}\right)^{\beta} \quad \text { with } \\
\beta & =\frac{\ln r n_{\text {mult }}}{\ln n_{\text {mult }}}=\frac{\ln r}{\ln n_{\text {mult }}}+1,
\end{aligned}
$$

which depends on the secondary particle multiplicity and the pion charge ratio of hadronic interactions. Moreover, the electron number at shower maximum can be estimated from

$$
N_{\text {max }, \mathrm{e}}=\frac{E_{\text {e.m. }}}{E_{\mathrm{c}}^{\text {e.m. }}}=\frac{E_{0}}{E_{\mathrm{c}}^{\text {e.m. }}}-N_{\mu} \frac{E_{\mathrm{c}}^{\pi}}{E_{\mathrm{c}}^{\mathrm{e} . \mathrm{m}} .},
$$

and is thus depending on the hadronic secondary multiplicity and pion charge ratio via the muon number. To relate the electron number at the shower maximum to the electron number at observation level, $N_{\mathrm{e}}$, it is necessary to take the strong attenuation of the electromagnetic shower component after the shower maximum into account. In the limit where $X_{\text {obs }} \gg X_{\max }$ the air shower size is in very good approximation exponentially attenuated with the scale length $\Lambda \sim 65 \mathrm{~g} / \mathrm{cm}^{2}$ [63]. We can thus use

$$
N_{\mathrm{e}} \sim N_{\mathrm{e}, \max } e^{-\left(X_{\mathrm{obs}}-X_{\max }\right) / \Lambda} \text { for } \quad X_{\mathrm{obs}} \gg X_{\max },
$$

which leads to

$$
\ln N_{\mathrm{e}} \sim \ln N_{\mathrm{e}, \max }-\left(X_{\mathrm{obs}}-X_{\max }\right) / \Lambda .
$$

It is important to note that $\ln N_{\mathrm{e}}$ depends only logarithmically on $N_{\mathrm{e}, \max }$ but linearly from $X_{\max }$. It is shown in Section $\nabla$ that indeed the impact of the depth of $X_{\max }$ on the electron number at ground level is dominating over a change following from Eq. (8). It is thus the strong longitudinal shower evolution that is responsible for the inability of the Heitler model to directly infer any dependence of $N_{\mathrm{e}}$ on hadronic interaction characteristics.

Within the hadronic Heitler framework it is not possible to follow the parallel development of the hadronic and electromagnetic cascades. To estimate the position of the shower maximum one is forced to consider the electromagnetic contribution from the first hadronic interaction only, which consists of $2 c n_{\text {mult }}$ e.m. subshowers each of the energy $E_{0} / n_{\text {mult }}$. The shower maximum is then

$$
X_{\max } \sim \lambda_{\mathrm{I}}+\lambda_{\mathrm{e}} \ln \frac{E_{0}}{n_{\text {mult }} E_{\mathrm{c}}^{\mathrm{e} . \mathrm{m}}},
$$

which is proportional to the hadronic interaction length and depends logarithmically on the multiplicity, but not on the charge ratio. This expression does not include the contribution to the electromagnetic cascade from the subsequent hadronic cascading process. However, the inclusion of higher hadronic generations does not change the structure of Eq. (10), only the coefficients change (e.g. [64]).

\section{Inelasticity in the Heitler Model}

In the Heitler model only equal energy particles of one type are considered. This also excludes any account of leading particle effects or other secondary particle distribution effects. To some limited extend it was accomplished to incorporate the inelasticity of interactions in a Heitler-type cascade by Matthews 22]. The inelasticity

$$
\kappa_{\text {inel }}=1-E_{\text {leading }} / E_{0}=1-\kappa_{\text {el }}
$$

is the fraction of the primary energy that is not carried away by the most energetic secondary particle, often referred to as leading particle. This energy fraction is available for the production of new secondary particles, mainly pions and kaons. The elasticity $\kappa_{\mathrm{el}}=1-\kappa_{\text {inel }}$ is the fraction of the primary energy that is carried by the leading particle.

The main difference compared to the standard Heitler model is that after each interaction secondaries with two energy levels are generated: $n_{\text {mult }}$ particles with energy of $\kappa_{\text {inel }} E_{0} / n_{\text {mult }}$ (of which $c n_{\text {mult }}$ are neutral pions) and one particle with energy of $\kappa_{\mathrm{el}} E_{0}$. So with an increasing number of hadronic generations the particles are distributed in more an more distinct energy levels. It turns out that in the generation $n$ there are in fact $n+1$ distinct groups of particles of identical energy. This situation is too complex to be handled in a compact analytic 
way. According to Matthews almost all secondary particles populate the one or two lowest energy levels per generation. Thus it can be justified to proceed as in the normal hadronic Heitler model and approximate the terminal hadronic generation $n_{\mathrm{c}}$ as where the average energy per pion drops below the critical energy, and thus

$$
n_{\mathrm{c}}=\frac{\ln \left(E_{0} / E_{\mathrm{c}}^{\pi}\right)}{\ln \left(\left(1+r n_{\text {mult }}\right) /\left(1-c \kappa_{\text {inel }}\right)\right)},
$$

where the total number of pions in generation $n$ is

$$
N_{n}^{\pi}=\left(1+r n_{\text {mult }}\right)^{n}
$$

and the average energy of these pions is

$$
E_{n}^{\pi}=E_{0}\left(1-c \kappa_{\text {inel }}\right)^{n} / N_{n}^{\pi} .
$$

The muon number is then

$$
\begin{aligned}
N_{\mu} & =\left(\frac{E_{0}}{E_{\mathrm{c}}^{\pi}}\right)^{\beta} \quad \text { with } \\
\beta & =\frac{\ln \left(1+r n_{\text {mult }}\right)}{\ln \left(\left(1+r n_{\text {mult }}\right) /\left(1-c \kappa_{\text {inel }}\right)\right)} \\
& =\left(1-\frac{\ln \left(1-c \kappa_{\text {inel }}\right)}{\ln \left(1+r n_{\text {mult }}\right)}\right)^{-1} .
\end{aligned}
$$

Since the inelasticity of hadronic interactions in air showers is of the order of 0.6 the approximation that the electromagnetic output of the first interaction is dominating the depth of the shower maximum is in fact not justified. Leading particles carry a large fraction of the total energy deeper into the atmosphere and contribute significantly to the electromagnetic cascade by finally generating e.m. sub-showers at larger depths. This superposition of electromagnetic subshowers starting at different hadronic generations does not fit into the analytical frame of the Heitler model, and thus no relation between inelasticity and $X_{\max }$ can be given. In fact, a simple extension of Eq. (10) suggests that the value of $X_{\max }$ would decrease proportional to $\lambda_{\mathrm{e}} \ln \left(1-\kappa_{\mathrm{el}}\right)$, while, on the contrary, it should increase with increasing elasticity because more energy is transferred deeper into the atmosphere. This is confirmed by our calculations, cf. Section $\nabla$. In all these considerations we have not taken into account the fact that the interaction cross sections, the elasticity, the secondary particle multiplicity and many more parameters of hadronic interactions depend on the energy. It is possible to improve the Heitler model to include some of these effects but this goes beyond the scope of this work (see, for example, 61]) .

In Table I the expected relations between features of hadronic interactions and important air shower observables are summarized. The results for $X_{\max }$ are all obtained from Eq. (10), where the relation $\lambda_{\mathrm{I}} \propto 1 / \sigma_{\mathrm{I}}$ is used. The results for the muon numbers are approximations of

$$
\ln N_{\mu}=\beta \ln \left(E_{0} / E_{\mathrm{c}}^{\pi}\right)
$$

TABLE I. Proportionalities found in the extended Heitler model between hadronic interaction features and the average of air shower observables. Some of the proportionalities are approximations that also depend on the value of other parameters.

\begin{tabular}{l||c|c|c} 
& $X_{\max }$ & $\ln N_{\mu}$ & $\ln N_{\mathrm{e}, \max }$ \\
\hline \hline hadr. cross section & $1 / \sigma_{\mathrm{I}}$ & 1 & 1 \\
multiplicity & $-\ln n_{\text {mult }}$ & $\sim-1 / \ln n_{\text {mult }}$ & $\sim 1 / \ln n_{\text {mult }}$ \\
elasticity & N/A & $\sim \kappa_{\mathrm{el}}$ & $\sim-\kappa_{\mathrm{el}}$ \\
pion charge ratio & 1 & $\sim-c$ & $\sim c$
\end{tabular}

based on Eq. (15). For comparison, Matthews quotes for constant $E_{0}$ that $\ln N_{\mu} \propto \beta \approx 1-0.14 \kappa_{\text {inel }}$, which corresponds to our result. The change of the electron numbers at the shower maximum is given by $\mathrm{d} \ln N_{\mathrm{e}, \max }=\mathrm{d} N_{\mathrm{e}, \max } / N_{\mathrm{e}, \max } \propto-\mathrm{d} N_{\mu} / N_{\mathrm{e}, \max }=$ $-N_{\mu} / N_{\mathrm{e}, \max } \mathrm{d} \ln N_{\mu} \propto-\mathrm{d} \ln N_{\mu}$, which is derived from Eq. (8). We will confront the results listed in this table with our simulations in Section $\mathrm{V}$

\section{Fluctuations of the Air Shower Development}

Not only the mean values of air shower observables are important, but also the fluctuations of these observables contain valuable information. They can complement the interpretation of air showers by exploiting different aspects of the shower development. Compared to average shower properties, the investigation of shower fluctuations has the potential to yield more reliable results.

The Heitler model has also some limited capabilities to explain the fluctuations of air shower cascades. According to Eq. (10) fluctuations in the depth of the shower maximum can be related to fluctuations of the depth of the first interaction $\mathrm{V}\left[X_{1}\right]=\lambda_{\mathrm{I}}^{2} \propto 1 / \sigma_{\mathrm{I}}^{2}$ and fluctuations

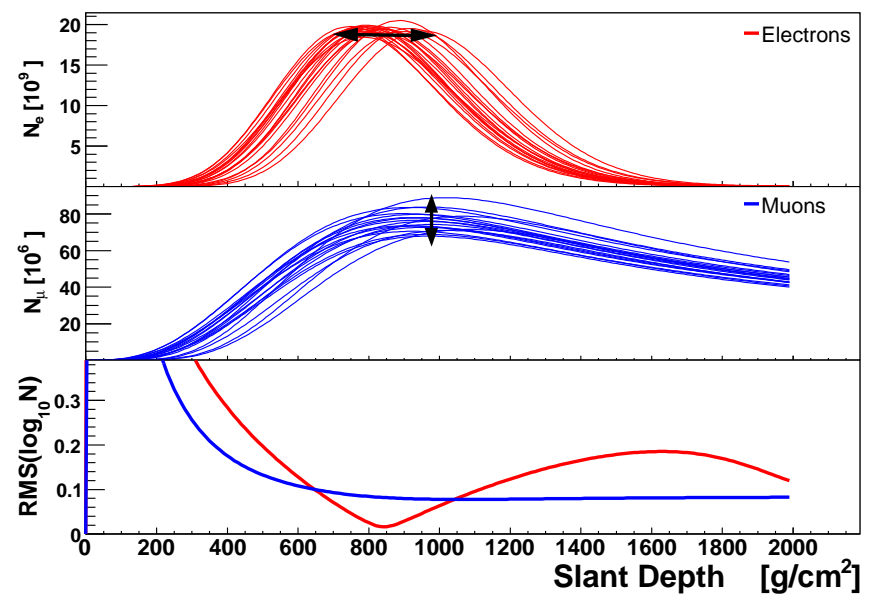

FIG. 5. Fluctuation of the longitudinal air shower development for 20 proton induced EAS at $E_{0}=10^{19.5} \mathrm{eV}$. 
of the multiplicity via

$$
\mathrm{V}\left[X_{\max }\right] \propto 1 / \sigma_{\mathrm{I}}^{2}+\lambda_{\mathrm{e}}^{2} \mathrm{~V}\left[\ln n_{\text {mult }}\right] .
$$

Thus, for small $\sigma_{\mathrm{I}}$, the fluctuations in $X_{\max }$ are depending on the cross section of the cosmic ray projectile in the atmosphere, while for very large $\sigma_{\mathrm{I}}$ the fluctuations of the multiplicity are dominating.

The fluctuations of the logarithm of the muon number can be derived from Eq. (16). The resulting coefficients are complicated functions of $c, k_{\text {inel }}, n_{\text {mult }}$ and $E_{\mathrm{c}}^{\pi}$, and it is not helpful to discuss them further. Fluctuations of the electron number are directly linked to the fluctuations of the muon number and $X_{\max }$ by Eqs. (8) and (9). It is demonstrated in Section $\mathbf{V}$ that the correlation between the fluctuations in the longitudinal shower development, i.e. the depth of the shower maximum, and the fluctuations of the electron number is very strong and mostly dominates over other effects.

To get an impression on important features of fluctuations of the longitudinal shower development of electron and muon number profiles, we perform some more detailed simulations beyond the Heitler model.

The characteristic fluctuations of air showers are demonstrated in Fig. 5. where electron and muon number profiles are shown for a series of equal energy air showers simulated with CONEX [35]. The depth of the electron number profiles shows large fluctuations while the electron number at the maximum is almost constant. On the other hand, the depth of the maximum in the muon profile does not show large fluctuations, but the maximum number of muons does. Also the electron profile has a very clear maximum with a fast absorption at larger depth, while the muon profile only exhibits a very moderate absorption after the maximum. This is also reflected in the logarithmic RMS of the electron and muon profiles around their mean profile. The electromagnetic part of the shower exhibit a very pronounced minimum in its fluctuations at the depth of the mean shower maximum $\left\langle X_{\max }\right\rangle$.

\section{SIMULATION OF AIR SHOWERS WITH MODIFIED INTERACTION MODELS}

In earlier studies of similar aim it was investigated how the interpretation of air showers is affected by changing internal parameters of individual hadronic interaction models [65 69] or by comparing the predictions of different interaction models 70 73. These approaches have the virtue of not, or only marginally, leaving the allowed parameter space of the original models. However, underlying assumptions that cannot be justified by fundamental theoretical principles, on which all models are built on, are often even more important than just parameter values. Because of this, only the phase-space in the direct vicinity of the original model can be explored by parameter variations. It is found that typically the differences between different models are larger than what can be obtained from variations of internal model parameters. Furthermore the available models do not exhaust the full range of current theoretical uncertainties.

This is why for our study we are not changing internal parameters of hadronic interaction models, but developed an ad hoc model to explore the uncertainties beyond the predictions of the available interaction models. Our model uses the predictions of existing hadronic event generators and modifies the output of these models in a suitable way to probe the phase-space of interaction characteristics more exhaustively. These modifications are ad hoc and explicitly not based on an underlying fundamental theory or phenomenology. This allows us, firstly, to apply our modification to any interaction model available and, secondly, to scan the phase-space of interaction physics very freely and extensively.

It is one of the advantages of our approach that it is not bound to parameter correlations normally arising from model-internal mechanisms. Therefore we can study the influence of the modification of one parameter on the shower evolution almost independently of the other parameters. For example, the increase of the inelasticity is typically correlated to an increase of the secondary particle multiplicity. This is not the case in our way of modifying the model predictions. Of course, energy and charge conservation imposes some correlation between different parameters that are present even in our approach.

\section{A. Considered Parameters of Hadronic Interactions}

We investigate the impact of the variation of several important features of hadronic interactions on typical air shower observables. These are

- the secondary multiplicity, $n_{\text {mult }}$, which is defined as the total number of secondaries produced in a hadronic collision,

- hadronic particle production cross sections, $\sigma_{\text {prod, }}$, that determine the interaction length of particles and thus the depth of the first interaction in the atmosphere, but also the speed of the development of the hadronic shower core,

- the elasticity, $\kappa_{\mathrm{el}}=E_{\text {leading }} / E_{\text {tot }}$ with $E_{\text {leading }}$ being the energy in the lab system carried by the leading particle after the collision and $E_{\text {tot }}$ the energy of the projectile in the lab system,

- and the pion charge-ratio, $c=n_{\pi^{0}} /\left(n_{\pi^{0}}+n_{\pi^{+}}+\right.$ $n_{\pi^{-}}$), with $n_{\pi^{\mathrm{x}}}$ the number of pions of type $\pi^{\mathrm{x}}$, which determines the fraction of particles going into the electromagnetic cascade of the air shower after each interaction, assuming that all $\pi^{0}$ decay basically instantly into $2 \gamma$.

The air shower observables that we study are the shower maximum, $X_{\max }$, the total number of electrons 


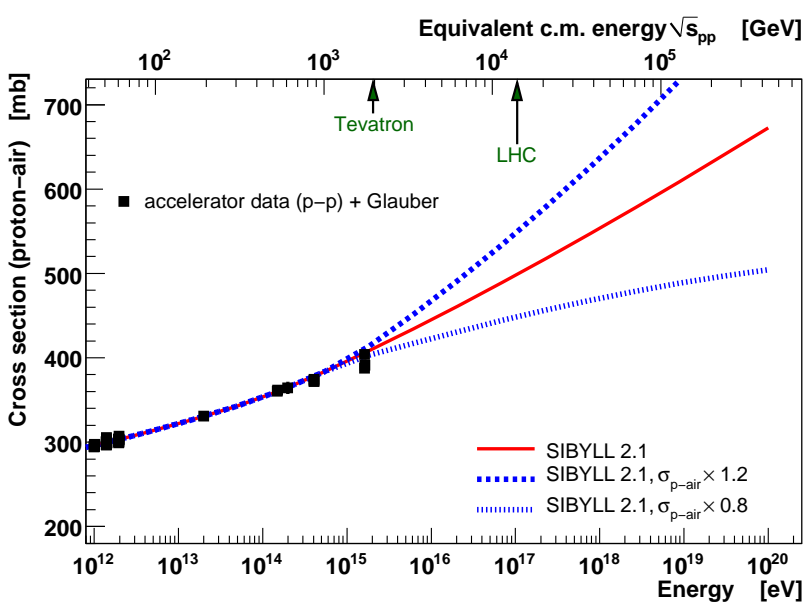

FIG. 6. Example of a modified cross section for SIBYLL for a $20 \%$ increase and decrease of $f_{19}$ (see [23, 28] for references to the data).

above $1 \mathrm{MeV}, N_{\mathrm{e}}$, as well as muons above $1 \mathrm{GeV}, N_{\mu}$, arriving at the slant depth of $X_{\mathrm{obs}}=1000 \mathrm{~g} / \mathrm{cm}^{2}$, and the invisible energy, $E_{\text {inv }}$, which is not accessible to calorimetric fluorescence telescope measurements.

\section{B. Extrapolation in Energy}

To investigate the importance of the extrapolation of high energy hadronic interaction models for the interpretation of EAS observables, we modified interaction characteristics during EAS simulations. To achieve this, individual hadronic interaction features are altered by the energy-dependent factor

$$
f\left(E, f_{19}\right)=1+\left(f_{19}-1\right) F(E)
$$

with

$$
F(E)=\left\{\begin{array}{ll}
0 & E \leq 1 \mathrm{PeV} \\
\frac{\log _{10}(E / 1 \mathrm{PeV})}{\log _{10}(10 \mathrm{EeV} / 1 \mathrm{PeV})} & E>1 \mathrm{PeV}
\end{array} .\right.
$$

The factor $f\left(E, f_{19}\right)$ is 1 below $10^{15} \mathrm{eV}$, where the models are constraint by accelerator data. Above $10^{15} \mathrm{eV}$, the deviation of $f\left(E, f_{19}\right)$ from 1 increases logarithmically with energy, reaching the value of $f_{19}$ at $10^{19} \mathrm{eV}$, cf. Fig. 6. This reflects the increasing uncertainty of the extrapolations with energy. The factor $f\left(E, f_{19}\right)$ is then used to re-scale specific characteristic properties of high energy hadronic interactions, such as the interaction cross section, secondary particle multiplicity etc.. Obviously, by doing this we rapidly leave the parameter space allowed by the original model; Thus, for large deviations from the original model the results are getting less reliable and have to be treated with some caution. Nevertheless, one can get a clear impression of how the resulting EAS properties are depending on the specific hadronic interaction property.

\section{Simulation of Showers}

As primary particles we will focus on proton and iron at an energy of $E_{0}=10^{19.5} \mathrm{eV}$. The elements in the mass range from proton to iron nuclei are the most abundant elements in cosmic rays. This way our results will bracket the expected mass range of the primary particles. An energy of about $10^{19.5} \mathrm{eV}$ is the highest energy at which one can expect to have good statistics of measured air showers with current and forthcoming observatories 74 , 75]. Above this energy the flux of cosmic rays is strongly suppressed [76, 77].

The simulations are mainly based on the interaction model Sibyll [34, 78, 79]. The choice of this model is not important for our studies as we will change the model output phenomenologically. It serves only as a reference for comparison with other models. We also performed cross checks with other interaction models for proton primaries.

All shower simulations are performed with the hybrid code CONEX [35]. The CONEX energy threshold above which particles are tracked individually is set to $10^{15} \mathrm{eV}$ to require full Monte Carlo simulation for all interactions above $10^{15} \mathrm{eV}$. The CONEX air shower simulation program was modified to evaluate $f\left(E, f_{19}\right)$ after each interaction of a hadronic particle (nucleon or meson) with a nucleus of air. In case $f\left(E, f_{19}\right) \neq 1$ a resampling algorithm [80] is applied to change the properties of final state particles of the corresponding interaction. The resampling algorithm is designed to specifically change only individual properties of the secondary particle distributions, while conserving all of the important features like total energy, charge, particle types, energy fractions in different particle types, and the leading particle as much as possible. For the detailed description of the resampling algorithms see Appendix A.

For primary cosmic ray nuclei with mass number $A>$ 1, the Glauber model [44, 45] is used to relate nucleus-air interactions to the underlying nucleon-air interactions. The hadronic event generator SIBYLL is based on the semi-superposition model 78], which allows us to apply the same resampling algorithms for showers of nuclear primaries. Thus, for primary cosmic ray nuclei other than protons we adopt the same modified SIBYLL interaction model but with each of the superimposed nucleonair interactions being individually changed according to $f\left(E, f_{19}\right)$-of course at the correspondingly lower energy of $E=E_{0} / A$ compared to the total energy of the projectile nucleus $E_{0}$. See Appendix $\mathrm{B}$ for details.

\section{RESULTS}

In the following all simulations are performed for proton and iron primaries of $10^{19.5} \mathrm{eV}$. Since the results discussed here are not very much depending on the particular choice of the primary energy, the findings are relevant to the analysis of air showers at least in the energy inter- 

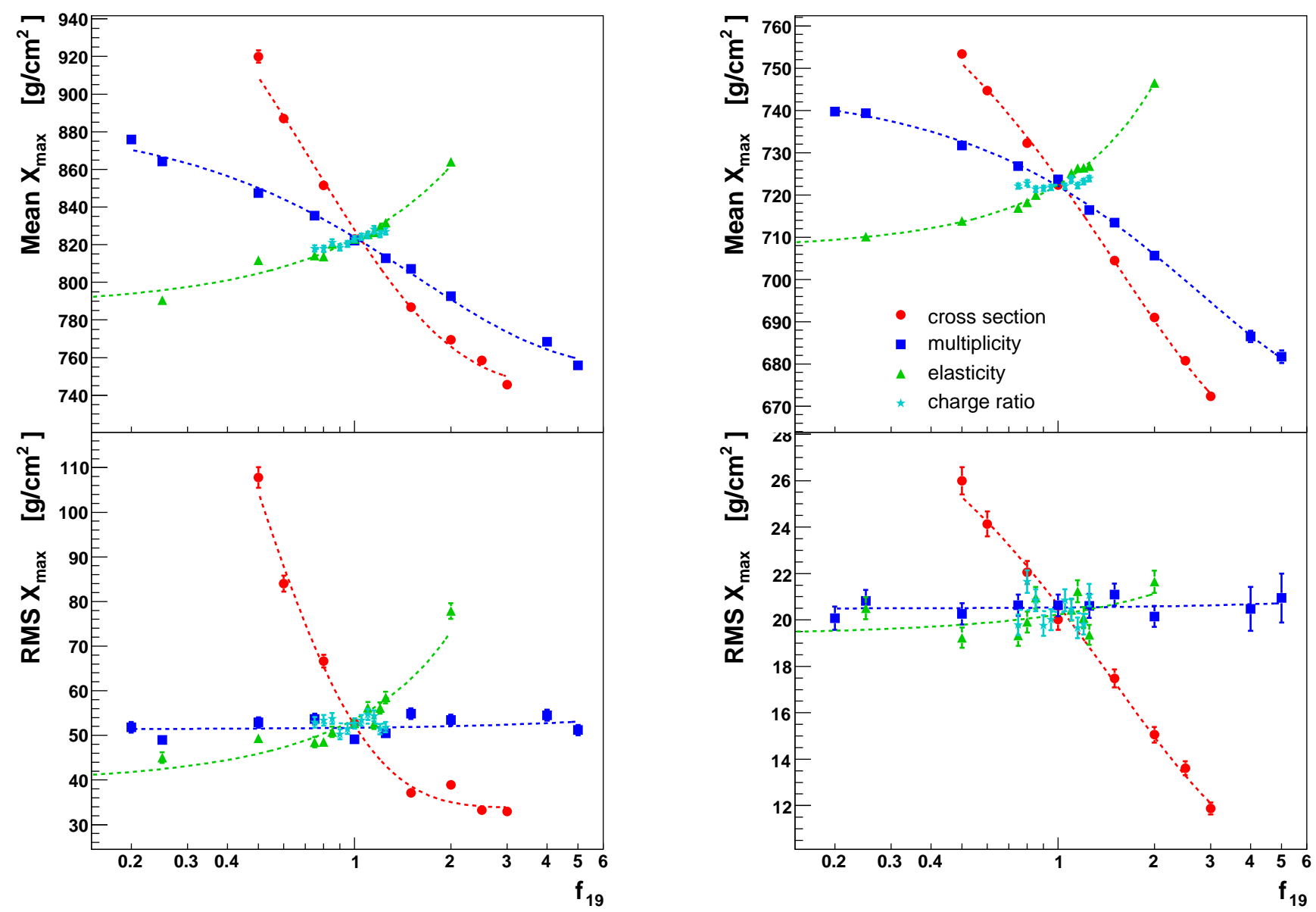

FIG. 7. Impact of hadronic interaction features on the shower maximum, $X_{\max }$, for proton (left) and iron (right) primaries.

val from $10^{19}$ to $10^{20} \mathrm{eV}$. For each point in the parameter space under investigation, 1000 showers are simulated.

In the discussion of our results we will frequently compare to the analytic Heitler model predictions summarized in Table I, and also refer to the dependence of EAS fluctuations on the longitudinal shower development as shown in Fig. 5 ,

\section{A. Longitudinal Shower Development and Depth of the Shower Maximum}

The results for the mean depth of shower maximum, $\left\langle X_{\max }\right\rangle$, and the fluctuation of $X_{\max }$, characterized by $\operatorname{RMS}\left(X_{\max }\right)$, are summarized in Fig. 7, The extrapolation of the total cross section for particle production has by far the biggest impact on $X_{\max }$. It can shift $\left\langle X_{\max }\right\rangle$ by almost $100 \mathrm{~g} / \mathrm{cm}^{2}$ for protons and $40 \mathrm{~g} / \mathrm{cm}^{2}$ for iron in both directions, and exhibits a strong correlation with the fluctuations of $X_{\max }$. All the other interaction characteristics considered here change the fluctuations only within a few $\mathrm{g} / \mathrm{cm}^{2}$, except the elasticity for proton pri- maries. A high elasticity leads to a moderate increase in fluctuations, at the same time shifting the $\left\langle X_{\max }\right\rangle$ deep into the atmosphere. The secondary multiplicity is almost as effective in shifting $\left\langle X_{\max }\right\rangle$ as the cross section. This is a consequence of the distribution of the same energy onto a growing number of particles, which is also predicted by the Heitler model. However, the dependence we find is somewhat different from the simple proportionality to $-\ln n_{\text {mult }}$ for larger deviations from the original model. For proton primaries the dependence on the cross section is similar to $1 / \sigma$ as in the Heitler model, especially at larger cross sections; For iron primaries, on the other hand, this change is more like $-\ln \sigma$. Furthermore, in contrary to the independence of $\left\langle X_{\max }\right\rangle$ from the pion charge ratio $c$ we find a slight trend $\propto \ln c$. The impact of the elasticity is approximately $\propto \kappa_{\mathrm{el}}$.

In addition to studying $X_{\max }$ we also considered the quantity $\Delta X=X_{\max }-X_{1}$, with $X_{1}$ being the depth of the first interaction in a shower. $\Delta X$ is only sensitive to the shower development that follows the first interaction. In Fig. 8 the results for $\Delta X$ are summarized.

As can be seen, only modifications of the cross section 

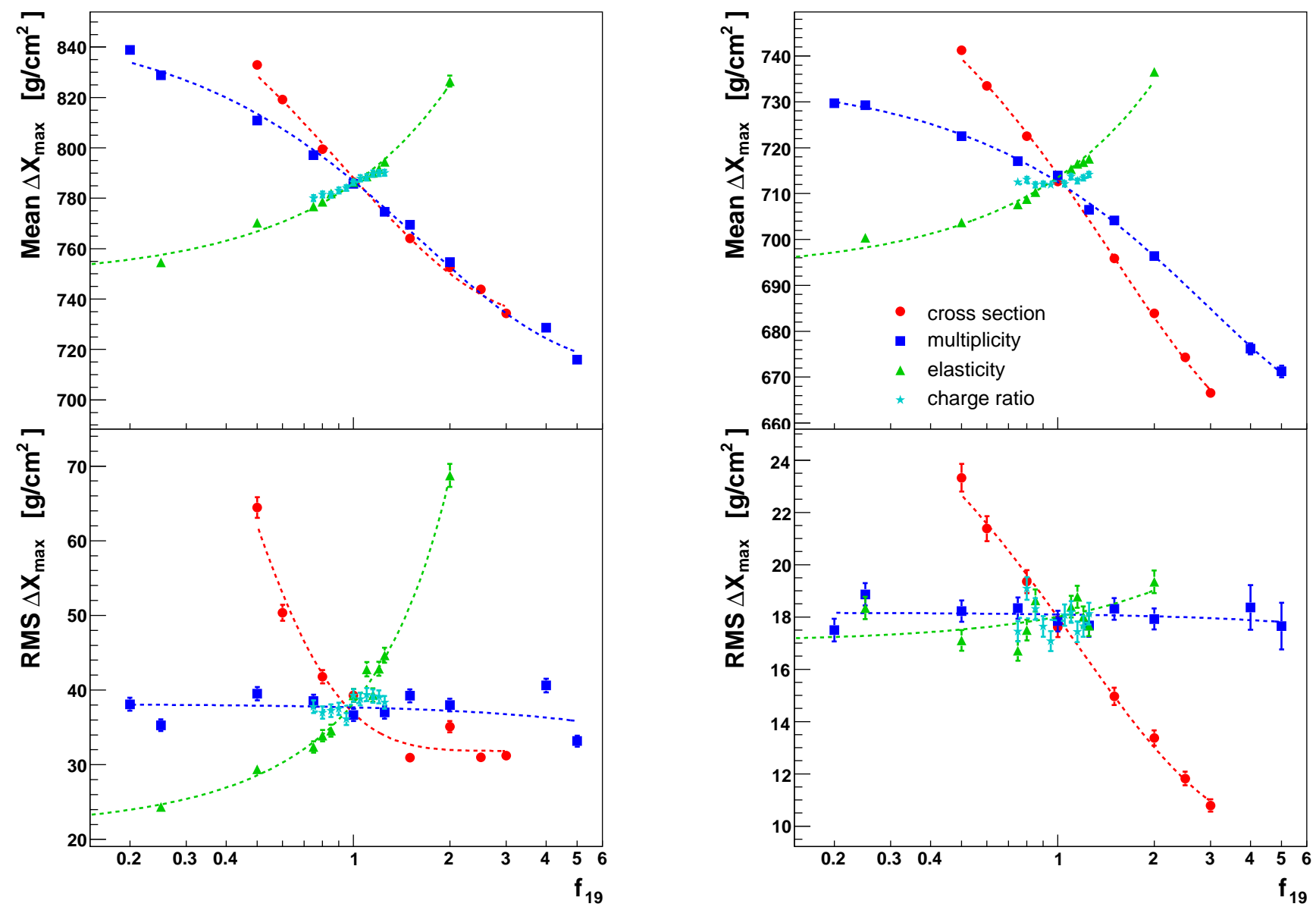

FIG. 8. Impact of the modification of hadronic interaction features on $\Delta X$ for proton (left) and iron (right) primaries.

change $X_{1}$ and $\Delta X$ at the same time. By construction, all other modifications have an identical impact on $X_{\max }$ and $\Delta X$, since they do not change $X_{1}$. Concerning the impact of the cross section extrapolation, we find that for iron primaries almost the full impact on $X_{\max }$ originates in fact from the air shower development after the first interaction $\Delta X$. The interaction length of iron in air at $10^{19.5} \mathrm{eV}$ is just $\sim 9 \mathrm{~g} / \mathrm{cm}^{2}$ while for protons it is $\sim$ $38 \mathrm{~g} / \mathrm{cm}^{2}$. Reducing the cross section by a factor of $f_{19}=$ 0.5 at $E=10^{19.5} \mathrm{eV}$ increases the interaction length by a factor of 1.3, which for iron accounts only for about $12 \mathrm{~g} / \mathrm{cm}^{2}$ of the total effect of $\sim 35 \mathrm{~g} / \mathrm{cm}^{2}$ seen in $\left\langle X_{\max }\right\rangle$. The rest is caused by the impact of a smaller cross section on the air shower development, and thus $\Delta X$. Even for proton primaries the air shower development contributes about half of the total effect on $\left\langle X_{\max }\right\rangle$. For example, lowering the cross section by a factor of 2 increases $\Delta X$ by $\sim 47 \mathrm{~g} / \mathrm{cm}^{2}$ while $X_{\max }$ changes by $\sim 97 \mathrm{~g} / \mathrm{cm}^{2}$.

For proton primaries the minimal possible fluctuation in $X_{\max }$ are about $\sim 35 \mathrm{~g} / \mathrm{cm}^{2}$. This asymptotic behavior of RMS $\left(X_{\max }\right)$ for large cross sections corresponds well to the predictions of the Heitler model, Eq. (17), where the asymptotic value of the fluctuations is related to fluctuations of the secondary multiplicity. For the case of iron primaries no such saturation effect is observed, which indicates a very much reduced importance of fluctuations induced by the secondary particle multiplicity. Also the much smaller overall dependence of $\operatorname{RMS}\left(X_{\max }\right)$ on the cross section for iron induced showers should be noted.

The independence of $\operatorname{RMS}\left(X_{\max }\right)$ of a modification of the multiplicity is related to $\mathrm{V}\left[X_{\max }\right] \propto \mathrm{V}\left[\ln n_{\text {mult }}\right]$ (c.f. Eq. (17)) and thus (with $f$ being the rescaling factor)

$$
\begin{gathered}
\operatorname{RMS}\left(X_{\text {max }}\right) \propto \mathrm{V}\left[\ln \left(f \cdot n_{\text {mult }}\right)\right]=\mathrm{V}[\ln f]+\mathrm{V}\left[\ln n_{\text {mult }}\right] \\
=\mathrm{V}\left[\ln n_{\text {mult }}\right] .
\end{gathered}
$$

A straightforward rescaling of the multiplicity, as performed by our ad hoc model, does not change the $X_{\max }$ fluctuations since the relative fluctuations of the multiplicity are unchanged.

The results shown in Fig. 7 can be used to gain some understanding of possible interpretations of existing data. For example, the fluctuations of $X_{\max }$ of $26 \pm 10$ (stat.) \pm 5 (syst.) $\mathrm{g} / \mathrm{cm}^{2}$ observed by the Pierre 

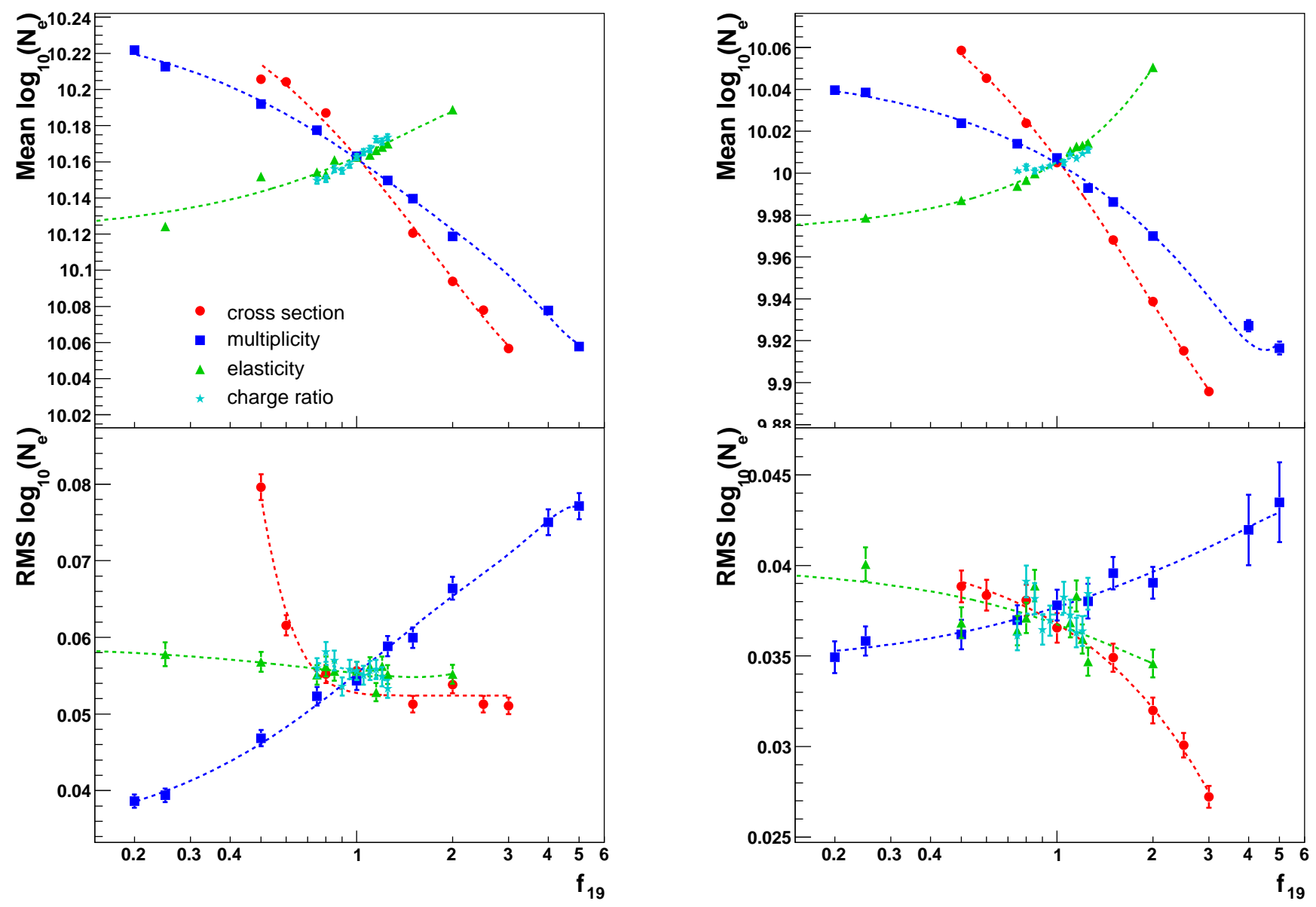

FIG. 9. Impact of a modified extrapolation of hadronic interaction features on the number of electrons, $N_{\mathrm{e}}$, for proton (left) and iron (right) primaries.

Auger Collaboration [8] at $10^{19.54} \mathrm{eV}$ are compatible at the $1 \sigma$ level with iron primaries, but also with proton primaries in combination with a strong increase of the cross section to higher energies. Of course, in addition to the $X_{\max }$ fluctuations also the mean depth of shower maximum and further observables have to be considered for obtaining a consistent interpretation of the data. Such an analysis is beyond the scope of this work.

\section{B. Electron Number at $X=1000 \mathrm{~g} / \mathrm{cm}^{2}$}

The impact of a modified extrapolation of hadronic interaction features on the electron number at $1000 \mathrm{~g} / \mathrm{cm}^{2}$ is shown in Fig. 9. The effect on the electron number is twofold. Firstly, there can be some direct influence of the interaction characteristics on $\log _{10} N_{\mathrm{e}}$. Secondly, and generally of even more importance, the change in the electron number reflects also the attenuation of the shower in the atmosphere: when $X_{\max }$ increases and gets closer to $1000 \mathrm{~g} / \mathrm{cm}^{2}$ the number of electrons increases, and when $X_{\max }$ decreases and veers away from the observation level, the number of electrons decreases. Therefore $\left\langle\log _{10} N_{\mathrm{e}}\right\rangle$ follows very much the corresponding trend in $\left\langle X_{\max }\right\rangle$, cf. Fig. 7 However, if the impact in $\log _{10} N_{\mathrm{e}}$ would be solely caused indirectly by $X_{\max }$ then we would e.g. expect the fluctuations to strictly follow the trend shown in Fig. 囵 which means fluctuations in $\log _{10} N_{\mathrm{e}}$ are minimal at $\left\langle X_{\max }\right\rangle$ and rapidly grow to smaller and larger depths. However, this trend is qualitatively found only for the modified multiplicity extrapolation. The elasticity, the charge-ratio and most clearly the cross section are not showing this behavior, or even an opposite effect. At the same time we also find that the influence of the multiplicity on $\left\langle\log _{10} N_{\mathrm{e}}\right\rangle$ is largest, and the importance of the extrapolation of the cross section, the elasticity, and the charge-ratio are reduced compared to that found for $X_{\max }$. All this is evidence that in addition to the indirect correlation by the longitudinal EAS development, the changes in the overall particle production within the air shower have a strong direct impact on the electron number. See Eq. (9) to get a qualitative description of 

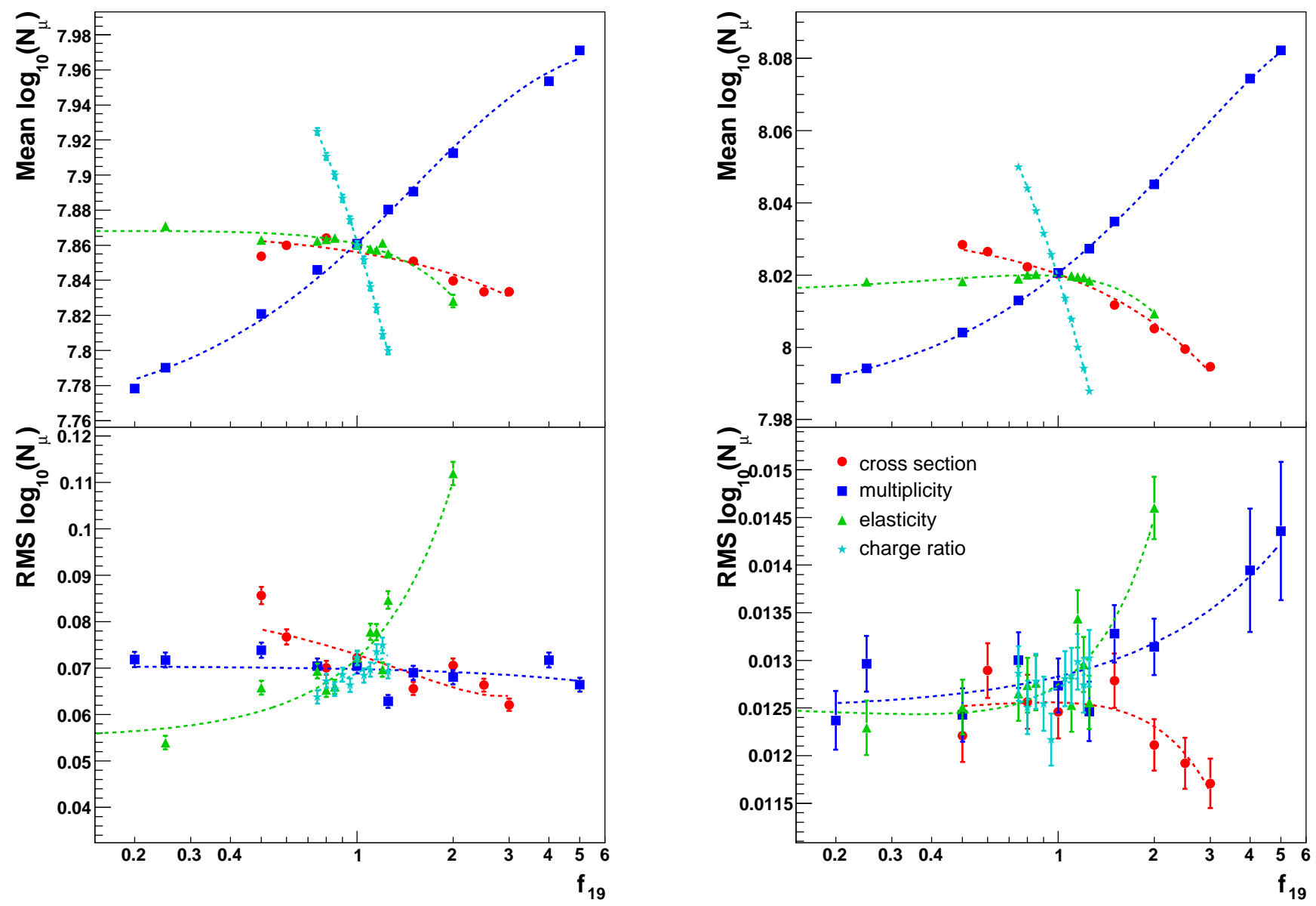

FIG. 10. Impact of a modified extrapolation of hadronic interaction features on the number of muons, $N_{\mu}$, for proton (left) and iron (right) primaries.

how $N_{\mathrm{e}, \max }$ and $X_{\max }$ are both affecting the electron number at ground level, $N_{\mathrm{e}}$. Note, that in Fig. 9 the limit $X_{\mathrm{obs}} \gg X_{\max }$ is not always fulfilled.

It is interesting to note, that an efficient way to change the fluctuations of the electron number in proton showers is via the extrapolation of the multiplicity, especially in the directions of reduced fluctuations. To increase the fluctuations, lowering the cross section can be equally effective. For iron primaries increasing the cross section leads to decreased fluctuations, while the overall impact of the multiplicity is very much reduced.

\section{Muon Number at $X=1000 \mathrm{~g} / \mathrm{cm}^{2}$}

The results of the influence of the modification of interaction features on the muon number are summarized in Fig. 10. In analogy to electrons also the muon number at $1000 \mathrm{~g} / \mathrm{cm}^{2}$ reacts to changes in the depth of $X_{\max }$ relative to the observation level. But as shown in Fig. 5. this sensitivity to $X_{\max }$ is much smaller than in the case of electrons. Especially the fluctuations are not having the clear minimum at $\left\langle X_{\max }\right\rangle$, but show a rather smooth transition to a very constant rate of fluctuations at larger depths. Furthermore, since muons in air showers are mainly produced via the decay of pions, their abundance is very sensitive to the overall number of pions in the shower. During the shower development there is a competition between pion decay, yielding muons and neutrinos, and interaction, producing new hadronic secondaries. While the hadronic interaction length of pions is $\lambda_{\text {int }} \sim 120 \mathrm{~g} / \mathrm{cm}^{2}$ over a wide range in energy, the decay length changes with energy $(\gamma=E / m)$ and is $\lambda_{\mathrm{dec}}=\rho \mathrm{c} \gamma \tau_{\mathrm{dec}}$. Muons are produced mainly by pions with $\lambda_{\text {dec }}<\lambda_{\text {int }}$. This is, for example, why high energy muons are more efficiently produced at high altitude where $\rho$ is small. When muons are produced, pions are removed from the multiplicative hadronic shower cascade and ultimately fewer pions in the air shower lead to the production of fewer muons. In addition, an increase of the production of high-energy muons at high altitude reduces the production of the more abundant muons at 

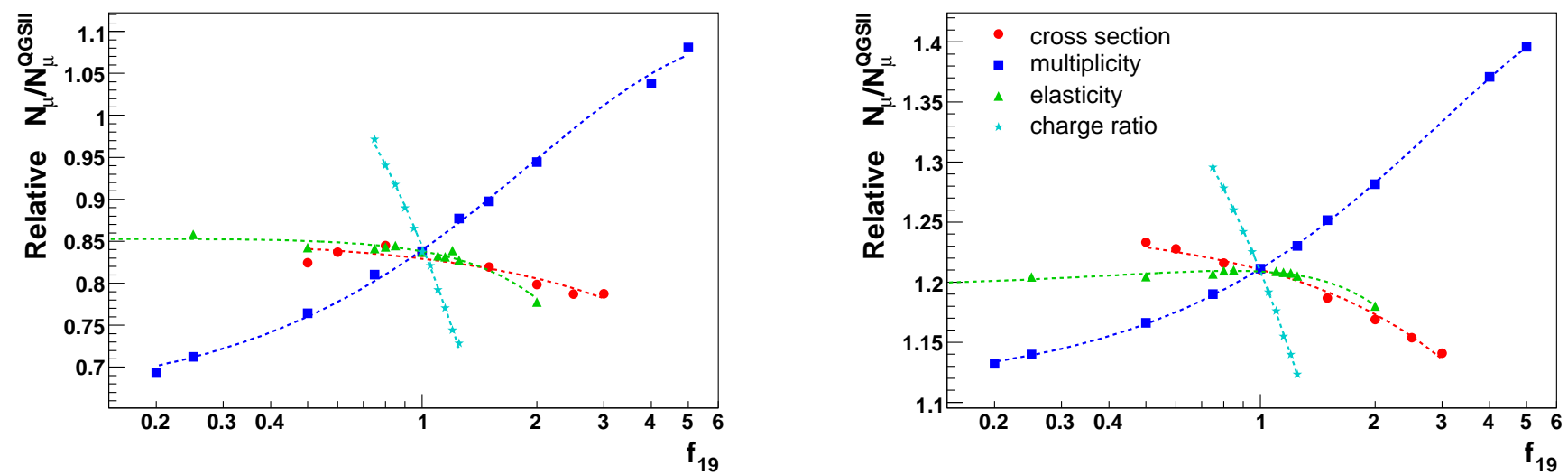

FIG. 11. Influence of the modification of hadronic interaction features on the number of muons relative to the prediction of protons/QGSJETII for proton (left) and iron (right) cosmic ray primaries.

lower energies.

Since muons are hardly attenuated in the atmosphere (cf. Fig. 51) the correlation of the muon number with the location of $X_{\max }$ is much reduced in comparison to the electron number. For muons the actual particle production characteristics in the EAS are much more directly important. This is demonstrated by the large positive correlation of $\left\langle\log _{10} N_{\mu}\right\rangle$ with the multiplicity, while for electrons a negative correlation is observed. In our simulations the muon number increases almost proportional to $\ln n_{\text {mult }}$. The Heitler model predicts a smaller effect of just $-1 / \ln n_{\text {mult }}$ at large multiplicities.

The muon number is also very sensitive to the chargeratio. A change in the charge-ratio is directly leading to a shift of the fractions of the primary energy going into the muonic and the electromagnetic shower components. The Heitler model indicates $\log _{10} N_{\mu} \propto-c$ and our results are consistent with this.

Similar to the case for the electron numbers the prediction by the Heitler model for the dependence on the elasticity is $\log _{10} N_{\mu} \propto \kappa_{\mathrm{el}}$, but our simulations show an opposite effect more like $\propto-\kappa_{\mathrm{el}}$. The fluctuation in $\log _{10} N_{\mu}$ for proton primaries depend mainly on the elasticity.

In Fig. 11] we show the change of the muon number obtained with the modified version of SIBYLL relative to the prediction of QGSJetII. Within the SibYLl model, the central parameters of the interaction have to be changed substantially to predict the same or even more muons than in QGSJETII. Only extreme modifications of the multiplicity or the charge ratio lead to an increase the muon number to the level of QGSJETII. However, one has to keep in mind that SiByLL is the model with the smallest muon numbers.

For example Epos 1.61, which predicts the highest muon numbers, yields up to $\sim 70 \%$ more muons than SiBYLl. This difference cannot be explained by a modification of the interaction features discussed here, whose change would lead to an increase of the muon number by only $\sim 30 \%$. As noted by the authors of Epos, a larger number of baryon-antibaryon pairs produced in this model keeps a larger fraction of the primary energy in the multiplicative hadronic shower component to eventually produce a significantly larger number of pions and thus muons at low energy [46]. Another important aspect is the chance probability of producing a neutral pion as a leading particle in charged pion interactions with air nuclei [81]. Differences in these features of hadronic interactions lead to large differences in the total number of muons predicted for air showers.

Recently the Pierre Auger Collaboration reported a measurement of the density of muons in showers of $10^{19} \mathrm{eV}$ at $1000 \mathrm{~m}$ from the core. Using proton induced showers as a reference, the number of muons is found to be about 1.3 times higher than that predicted by using QGSJETII for high energy and FLUKA for low energy interactions [7]. Our studies show that increasing the total muon number in showers by $30 \%$ requires, for a given primary composition, rather drastic changes of the features of the hadronic interactions considered in this work. In contrast, already moderate modifications of the modelling of low energy interactions lead to a change of the muon number of this order, see discussion in [62, 82, 83]. A good knowledge of particle production is needed both at high and low energy to be able to use the muon number of showers for absolute measurements of the mass of the primary particle.

\section{Invisible Energy}

It is one of the advantages of the observation of air showers by fluorescence telescopes that the measured signal is proportional to the energy deposit $\mathrm{d} E / \mathrm{d} X$ of the 

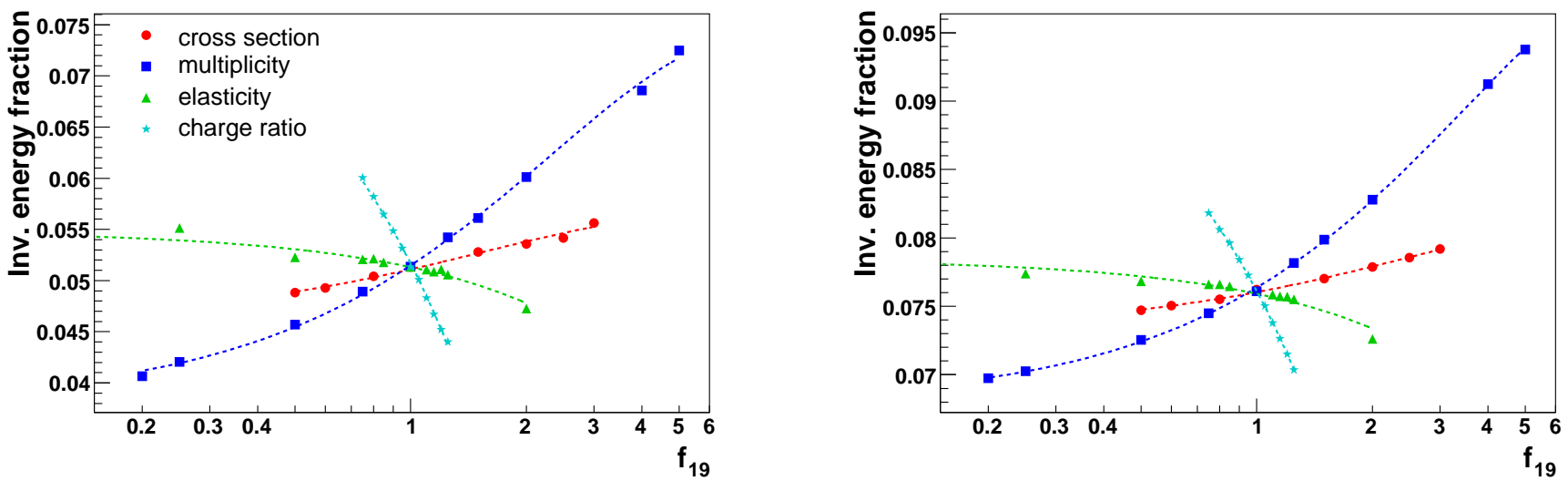

FIG. 12. Impact of modified extrapolations of interaction features on the invisible energy fraction, $\epsilon_{\text {inv }}$, for proton (left) and iron (right) primaries.

air shower in the atmosphere [18, 84, 85]. The integral

$$
E_{0}^{\text {e.m. }}=\int \mathrm{d} X \frac{\mathrm{d} E}{\mathrm{~d} X}(X)=\int \mathrm{d} X \alpha_{\mathrm{eff}}(X) N_{\mathrm{e}}(X)
$$

where $\alpha_{\text {eff }}$ is the effective (i.e. average) energy loss per electron, yields then an accurate measurement of the total energy of the primary cosmic ray particle that was transferred to the electromagnetic shower component. Muons and hadrons are much less abundant compared to electrons and also ionize less efficiently, and neutrinos do not deposit any energy. The invisible energy fraction 85. is

$$
\begin{aligned}
\epsilon_{\text {inv }} & =\left(E_{0}-E_{0}^{\text {e.m. }}\right) / E_{0} \quad \text { and thus } \\
E_{0} & =E_{0}^{\text {e.m. }} /\left(1-\epsilon_{\text {inv }}\right)
\end{aligned}
$$

cannot be calculated in a purely model independent way, since $\epsilon_{\text {inv }}$ has to be estimated from air shower simulations. Fortunately $\epsilon_{\mathrm{inv}}$ is only of the order of a few percent (see Fig. 12) and thus the introduced model dependence of the estimated total shower energy is small.

The invisible energy fraction is naturally correlated to the number of muons in the air shower. The trends we observe are indeed very similar to what is seen for muon numbers. However, the impact on $\epsilon_{\mathrm{inv}}$ is generally larger than for $N_{\mu}$, indicating the importance of additional effects like enhanced muon production and differing energy distribution of electrons or muons. Most strikingly the small trend to smaller muon numbers of a rising cross section is reversed into a trend to larger $\epsilon_{\mathrm{inv}}$. A large cross section causes an accelerated shower development. The rise of the invisible energy is related to an increasing number of high energy muons produced high up in the atmosphere.

Our simulations indicate that, over the full parameter range of the modifications, the invisible energy fraction does not change by more than $\sim 0.02$.

\section{DEPENDENCE ON THE INTERACTION MODEL}

So far all the calculations were based on SIBYLL 2.1. We will now present a comparison of the previously discussed results with that obtained with the models QGSJet01C, QGSJetII.3 and Epos 1.61. This study is limited to the case of proton primaries, since the Glauber model used for iron primaries does not fit into the framework of any of these models.

We find that the impact of the changes of the extrapolation of hadronic multiparticle production on air shower predictions is very similar for all models. Only EPOS 1.61 is found to behave somewhat different. The most striking difference between EPOs and the other models is found for $\operatorname{RMS}\left(X_{\max }\right)$, see Fig. 13, All model predictions seem to be virtually independent of the multiplicity, only EPOS shows a clear trend to increased shower fluctuations for higher multiplicities. Also the correlation of the elasticity and $\operatorname{RMS}\left(X_{\max }\right)$ is stronger in EPOS in comparison to the other models. Furthermore, an increase of the cross section within Epos does not lead to a significant reduction of the shower fluctuations in $X_{\max }$. This indicates a different contribution of the residual fluctuations caused by the secondary multiplicity in EPOs than in the other models (c.f. Eq. (17)).

The muon number itself is known to be one of the shower observables with the largest inherent modelling uncertainties. At the same time it is an excellent example of how model-independent the relation between modifications of the interaction characteristics to air shower observables is, see Fig. 14. While the underlying large differences in the model predictions are clearly visible, the dependence on the modifications is very similar. The largest differences can be observed for the elasticity where, similar to the case of $\operatorname{RMS}\left(X_{\max }\right)$, the effect in EPOS is larger than for all other models. 

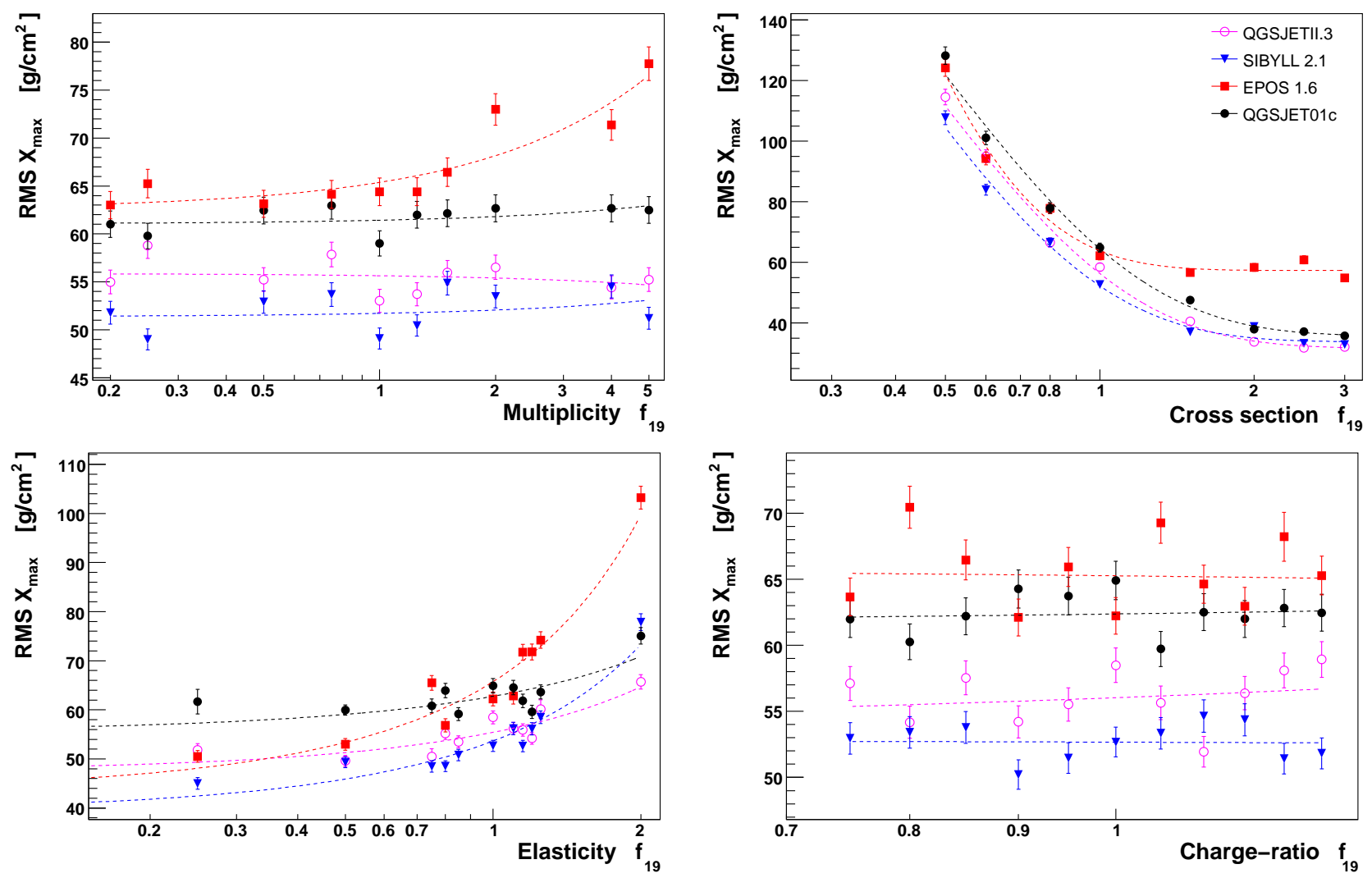

FIG. 13. Influence of modified extrapolations of particle production on $\operatorname{RMS}\left(X_{\max }\right)$ for several hadronic interaction models.
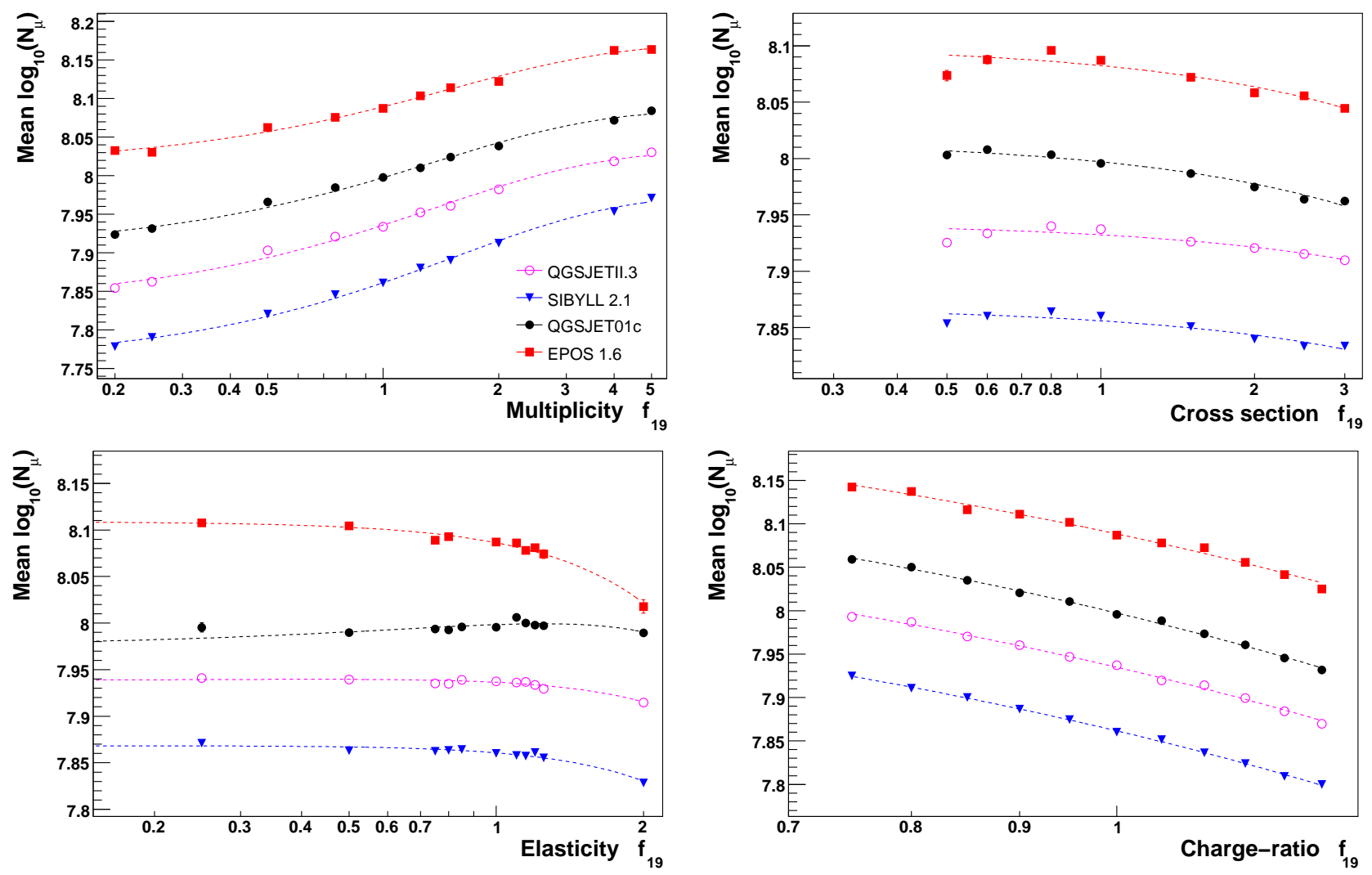

FIG. 14. Impact of modifications of the extrapolation of particle production on $\left\langle\log _{10} N_{\mu}\right\rangle$ for several hadronic interaction models. 


\section{CONCLUSION AND OUTLOOK}

We developed an ad hoc model to modify features of hadronic interaction in the simulation of extensive air showers. The algorithms for these modifications were tested using high statistics data sets of simulated p-air interactions at fixed energy. We then used this ad hoc model to explore the impact of different extrapolations of hadronic interaction characteristics on the predictions for air shower development and typical air shower observables.

For simplicity it is assumed that the properties of hadronic interactions are sufficiently well known at energies below $10^{15} \mathrm{eV}$. Only the extrapolation of these features to higher energies are changed, while ensuring a steady transition of the description of hadronic particle production from low to high energy.

Our study is mainly based on the interaction model Sibyll 2.1. This choice is driven by the fact that our model for introducing modifications to nucleus-air interactions is founded on the Glauber model and the semi-superposition model as implemented in SIBYLL. The dependence of the results on the particular interaction model is small. Cross checks with QGSJET01C, QGSJETII.3, and EPOS 1.61 show that predictions for air showers change consistently in dependence on the modification we introduce for hadronic interactions. Some deviations from a universal behaviour of the models is found for Epos, being most likely related to the qualitatively different structure of this model.

Many of the observed dependences of air shower predictions on features of hadronic multiparticle production can be understood qualitatively within the simple Heitler model of particle cascades and its extension by Matthews. The most relevant results on the impact of different extrapolations of hadronic interaction features on air shower observables are

- The longitudinal air shower fluctuations, as described by $\operatorname{RMS}\left(X_{\max }\right)$, depend mainly on the cross section and less strongly on the elasticity. This makes fluctuations in $X_{\max }$ a good parameter to study hadronic cross sections at ultra-high energies.

- The electron number is negatively correlated with the multiplicity, whereas the muon number shows a positive correlation. The secondary particle multiplicity provides a powerful handle to change the electron vs. muon number ratio in air showers.

- The invisible energy fraction is affected by at most \pm 0.02 by uncertainties of the extrapolation of hadronic interaction features to air shower energies.

- To increase the muon number in air showers the multiplicity of interactions can be increased, or the charge-ratio decreased. A change of the predicted muon number in air showers of more than $30 \%$ seems almost impossible to obtain by only modifying high energy interactions in the simulation as described here.

It turns out that the influence of the extrapolation of hadronic interaction features on air shower observables and the dependence of these observables on the mass of the primary particle are of similar magnitude. Therefore it is very difficult to use shower measurements to estimate the characteristics of hadronic multiparticle production at ultra-high energy. External knowledge on the primary mass composition will be needed for reliable estimates. Such information could come from models of the sources of ultra-high energy cosmic rays or the observation of magnetic deflection of the particles in the Galaxy. In addition the suppression of the cosmic ray flux above $10^{19.7} \mathrm{eV}$ offers a unique window to a monoor bi-elemental cosmic ray beam: almost only proton or iron particles are expected to arrive at Earth [86, 87] at ultra-high energy from distant sources.

The measurement of hadronic multiparticle production at LHC energy has the potential to significantly reduce the spread of the model divergences at cosmic ray energies. If the information most relevant to the air shower development will be extracted from the LHC data, this will very likely lead to a breakthrough in the analysis of air shower data. Already now experiments at the LHC measured multiplicity pseudorapidity distributions in the central event region very accurately. However, the really important measurements for the understanding of air shower cascades will be: leading particle effects, multiplicities in forward direction and hadronic cross sections. These measurements are very difficult to perform-mostly because they require to measure the very forward directed particle production-and will thus take much more time to be measured to high precision.

\section{ACKNOWLEDGMENTS}

The authors want to thank their colleagues from the Pierre Auger Collaboration, and especially Prof. P. Sommers and Dr. T. Pierog, for numerous discussions. The simulations needed for this work were performed at the Campus-Grid [88] at the Steinbuch Centre for Computing of the KIT. This work has been supported in part by Bundesministerium für Bildung und Forschung (BMBF) grant No. 05A08VK1.
[1] J. Abraham et al. (Pierre Auger), Nucl. Instrum. Meth. A523, 50 (2004).
[2] H. Kawai et al. (TA), Nucl. Phys. Proc. Suppl. 
175-176, 221 (2008).

[3] R. Abbasi et al. (HiRes), Astrophys. J. 622, 910 (2005), astro-ph/0407622

[4] B. R. Dawson, R. Meyhandan, and K. M. Simpson, Astropart. Phys. 9, 331 (1998), astro-ph/9801260.

[5] M. Ave et al., Astropart. Phys. 19, 61 (2003), astro-ph/0203150

[6] M. T. Dova, M. E. Mancenido, A. G. Mariazzi, T. P. McCauley, and A. A. Watson, Astropart. Phys. 21, 597 (2004), arXiv:astro-ph/0312463

[7] J. Abraham et al. (Pierre Auger), Proc. of $31^{\text {th }}$ Int. Cosmic Ray Conf., Lodz, Poland(2009), arXiv:0906.2319 [astro-ph]

[8] J. Abraham et al. (Pierre Auger), Phys. Rev. Lett. 104, 091101 (2010), arXiv:1002.0699 [astro-ph].

[9] T. Antoni et al. (KASCADE), Astropart. Phys. 24, 1 (2005), astro-ph/0505413

[10] M. Nagano et al., J. Phys. G18, 423 (1992).

[11] M. Takeda et al., Phys. Rev. Lett. 81, 1163 (1998), astro-ph/9807193.

[12] M. Ave, J. Knapp, J. Lloyd-Evans, M. Marchesini, and A. A. Watson, Astropart. Phys. 19, 47 (2003), astro-ph/0112253

[13] M. Aglietta et al. (EAS-TOP), Astropart. Phys. 21, 583 (2004).

[14] M. Amenomori et al. (Tibet AS $\gamma$ ), Astrophys. J. 678, 1165 (2008), arXiv:0801.1803 [hep-ex]

[15] D. Heck, "The influence of hadronic interaction models on simulated air-showers: A phenomenologic comparison," (2008), talk given at CORSIKA School 2008, Freudenstadt, Germany, 25 - 30 November 2008, available at http://www-ik.fzk.de/corsika/corsika-school2008/.

[16] B. Rossi and K. Greisen, Rev. Mod. Phys. 13, 240 (1941).

[17] M. Giller, A. Kacperczyk, J. Malinowski, W. Tkaczyk, and G. Wieczorek, J. Phys. G31, 947 (2005).

[18] F. Nerling, J. Blümer, R. Engel, and M. Risse, Astropart. Phys. 24, 421 (2006).

[19] F. Schmidt, M. Ave, L. Cazon, and A. S. Chou, Astropart. Phys. 29, 355 (2008), arXiv:0712.3750 [astro-ph]

[20] P. Lipari, Phys. Rev. 79, 063001 (2008), arXiv:0809.0190 [astro-ph]

[21] S. Lafebre et al., Astropart. Phys. 31, 243 (2009), arXiv:0902.0548 [astro-ph.HE]

[22] J. Matthews, Astropart. Phys. 22, 387 (2005).

[23] R. Engel, Nucl. Phys. Proc. Suppl. 82, 221 (2000).

[24] A. Donnachie and P. Landshoff, Phys. Lett. B296, 227 (1992), hep-ph/9209205

[25] G. Pancheri, R. M. Godbole, A. Grau, and Y. N. Srivastava, Acta Phys. Polon. B38, 2979 (2007), arXiv:hep-ph/0703174

[26] P. Landshoff, Lectures at School on QCD, Calabria(July 2007), arXiv:0709.0395 [hep-ph].

[27] P. V. Landshoff(2009), arXiv:0903.1523 [hep-ph]

[28] R. Ulrich, R. Engel, S. Muller, F. Schussler, and M. Unger, Nucl. Phys. Proc. Suppl. 196, 335 (2009), arXiv:0906.3075 [astro-ph]

[29] S. Ostapchenko, Nucl. Phys. Proc. Suppl. 151, 143 (2006), hep-ph/0412332.

[30] S. Ostapchenko, Phys. Lett. B636, 40 (2006), hep-ph/0602139

[31] K. Werner and T. Pierog, AIP Conf. Proc. 928, 111
(2007), arXiv:0707.3330 [astro-ph]

[32] N. Kalmykov and S. Ostapchenko, Phys. Atom. Nucl. 56, 346 (1993).

[33] N. Kalmykov and S. Ostapchenko, Sov. J. Nucl. Phys. 50, 315 (1989).

[34] E.-J. Ahn, R. Engel, T. K. Gaisser, P. Lipari, and T. Stanev, Phys. Rev. D80, 094003 (2009), arXiv:0906.4113 [hep-ph].

[35] T. Bergmann et al., Astropart. Phys. 26, 420 (2007), astro-ph/0606564

[36] H. Drescher, M. Hladik, S. Ostapchenko, T. Pierog, and K. Werner, Phys. Rept. 350, 93 (2001), hep-ph/0007198

[37] M. Hladik, H. Drescher, S. Ostapchenko, T. Pierog, and K. Werner, Phys. Rev. Lett. 86, 3506 (2001), hep-ph/0102194

[38] J. N. Capdevielle, J. Phys. G15, 909 (1989).

[39] J. Ranft, Phys. Rev. D51, 64 (1995).

[40] K. Werner, Phys. Rep. 232, 87 (1993).

[41] D. Heck et al., Proc. of 27th Int. Cosmic Ray Conf., Hamburg 1, 233 (2001).

[42] T. Antoni et al. (KASCADE), Astropart. Phys. 16, 245 (2002), astro-ph/0102443

[43] T. Abu-Zayyad et al. (HiRes-MIA), Phys. Rev. Lett. 84, 4276 (2000), astro-ph/9911144

[44] R. Glauber, Phys. Rev. 100, 242 (1955).

[45] R. Glauber and G. Matthiae, Nucl. Phys. B21, 135 (1970).

[46] T. Pierog and K. Werner, Proc. of $30^{\text {th }}$ Int. Cosmic Ray Conf., Merida, Mexico(2007), astro-ph/0611311.

[47] T. Pierog and K. Werner, Proc. of 30th Int. Cosmic Ray Conf., Merida 4, 629 (2007).

[48] K. Aamodt et al. (ALICE), Phys. Rev. Lett. 105, 072002 (2010), arXiv:1006.5432 [hep-ex]

[49] K. Aamodt et al. (ALICE), Eur. Phys. J. C68, 89 (2010), arXiv:1004.3034 [hep-ex]

[50] K. Aamodt et al. (ALICE), Eur. Phys. J. C68, 345 (2010), arXiv:1004.3514 [hep-ex]

[51] K. Aamodt et al. (ALICE)(2009), arXiv:0911.5430 [hep-ex]

[52] G. Aad et al. (ATLAS), Phys. Lett. B688, 21 (2010), arXiv:1003.3124 [hep-ex]

[53] V. Khachatryan et al. (CMS), JHEP 02, 041 (2010), arXiv:1002.0621 [hep-ex]

[54] V. Khachatryan et al. (CMS), Phys. Rev. Lett. 105, 022002 (2010), arXiv:1005.3299 [hep-ex]

[55] V. Khachatryan et al. (CMS)(2010), arXiv:1006.2083 [hep-ex]

[56] D. d'Enterria, R. Engel, S. Ostapchenko, T. Pierog, and K. Werner(2010), in preparation.

[57] For a historical account of the development of this model see [89].

[58] W. Heitler, Rev. Mod. Phys. 21, 113 (1949).

[59] T. Pierog, R. Engel, and D. Heck, Czech. J. Phys. 56, A161 (2006), astro-ph/0602190.

[60] T. K. Gaisser, Cosmic rays and particle physics (Cambridge Univ. Press, Cambridge, 1990).

[61] J. Alvarez-Muniz, R. Engel, T. K. Gaisser, J. A. Ortiz, and T. Stanev, Phys. Rev. D66, 033011 (2002), astro-ph/0205302

[62] C. Meurer, J. Blümer, R. Engel, A. Haungs, and M. Roth, Czech. J. Phys. 56, A211 (2006), astro-ph/0512536

[63] It is a feature of air shower universality (see, for 
example, 16 21]) that $\Lambda$ does not depend on the primary particle or hadronic interaction model.

[64] J. Alvarez-Muniz, R. Engel, T. Gaisser, J. Ortiz, and T. Stanev, Phys. Rev. D66, 033011 (2002), astro-ph/0205302.

[65] J. Bellandi, R. J. M. Covolan, and A. L. Godoi, Phys. Lett. B343, 410 (1995).

[66] R. Engel, Nucl. Phys. Proc. Suppl. 122, 40 (2003).

[67] S. S. Ostapchenko, J. Phys. G29, 831 (2003).

[68] S. Ostapchenko, Czech. J. Phys. 56, A149 (2006), arXiv:hep-ph/0601230.

[69] C. Bleve, R. Parsons, J. Knapp, and S. Ostapchenko, Proc. of $31^{\text {th }}$ Int. Cosmic Ray Conf., Lodz, Poland(2009).

[70] J. Knapp, D. Heck, and G. Schatz, "Comparison of hadronic interaction models used in air shower simulations and of their influence on shower development and obsevables," (1996), in Wissenschaftliche Berichte FZKA 5828, Forschungszentrum Karlsruhe.

[71] J. Knapp, D. Heck, S. Sciutto, M. Dova, and M. Risse, Astropart. Phys. 19, 77 (2003), astro-ph/0206414.

[72] L. A. Anchordoqui, M. T. Dova, L. N. Epele, and S. J. Sciutto, Phys. Rev. D59, 094003 (1999), arXiv:hep-ph/9810384.

[73] C. A. Garcia Canal, S. J. Sciutto, and T. Tarutina, Phys. Rev. D79, 054006 (2009), arXiv:0903.2409 [astro-ph.HE]

[74] J. Blümer et al. (Pierre Auger), New J. Phys. 12, 035001 (2010).

[75] A. Santangelo and A. Petrolini, New J. Phys. 11, 065010 (2009).

[76] R. Abbasi et al. (HiRes), Phys. Rev. Lett. 100, 101101 (2008), astro-ph/0703099

[77] J. Abraham et al. (Pierre Auger), Phys. Rev. Lett. 101, 061101 (2008), arXiv:0806.4302 [astro-ph]

[78] J. Engel, T. Gaisser, T. Stanev, and P. Lipari, Phys. Rev. D46, 5013 (1992).

[79] R. Fletcher, T. Gaisser, P. Lipari, and T. Stanev, Phys. Rev. D50, 5710 (1994).

[80] Available upon request from ralf.ulrich@kit.edu.

[81] H.-J. Drescher, Phys. Rev. D77, 056003 (2007), arXiv:0712.1517 [hep-ph]

[82] H.-J. Drescher, M. Bleicher, S. Soff, and H. Stoecker, Astropart. Phys. 21, 87 (2004), astro-ph/0307453.

[83] I. C. Maris et al., Nucl. Phys. Proc. Suppl. 196, 86 (2009), arXiv:0907.0409 [astro-ph.CO]

[84] C. Song et al. (HiRes), Astropart. Phys. 14, 7 (2000), astro-ph/9910195.

[85] H. M. J. Barbosa, F. Catalani, J. A. Chinellato, and C. Dobrigkeit, Astropart. Phys. 22, 159 (2004), arXiv:astro-ph/0310234.

[86] D. Harari, S. Mollerach, and E. Roulet, JCAP 0611, 012 (2006), arXiv:astro-ph/0609294.

[87] D. Allard, N. G. Busca, G. Decerprit, A. V. Olinto, and E. Parizot, JCAP 0810, 033 (2008), arXiv:0805.4779 [astro-ph]

[88] www.campusgrid.de.

[89] J. Linsley, Sci. Am. 239, 48 (1978). 


\section{Appendix A: Modified Characteristics of Hadronic Interactions}

The CONEX air shower simulation program was modified allowing us to change specific properties of the particle production characteristics of the underlying high energy event generators.

The resampling algorithms are written in order to specifically change individual properties of the secondary particle distributions, while conserving all of the important features like total energy, charge, particle types, energy fractions in different particle types and the leading particle as far as possible. Since CONEX is a 1DEAS simulation code, no special efforts are made to conserve $p_{\perp}$. If the energy $E$ of a secondary particle is altered to yield $E_{\text {mod }}$ its momentum $\vec{p}$ is re-computed as $\vec{p}_{\text {mod }}=\vec{p} \sqrt{E_{\text {mod }}^{2}-m^{2}} /|\vec{p}|$.

The algorithms are applied to the secondary particles of interactions in the laboratory frame of the air shower. This is the frame that is most relevant for the air shower development, and i.e. conservation of the total energy is paramount within this frame.

In the following we will describe in detail how the algorithms work to achieve the modification of specific interaction features. All algorithms are controlled by the energy dependent modification factor $f\left(E, f_{19}\right)$, see Eqs. (18) and (19).

For the discussion we use the quantities pseudorapidity $\eta=-\ln \tan \theta / 2, X_{\text {lab }}=E / E_{0}$ and the number of charged secondaries, $N_{\mathrm{ch}}$.

\section{Cross Sections}

Modifying the cross section can be implemented straightforwardly. No secondary particle resampling is needed. Only the extrapolated value of all hadronic cross sections has to be multiplied by $f\left(E, f_{19}\right)$

$$
\sigma^{\text {mod }}=\sigma^{\text {orgi }} f\left(E, f_{19}\right) .
$$

See Fig. 6 for an example of this modification. The rescaling is not only done for the primary particle, and hence $\sigma_{\mathrm{p}-\mathrm{air}}$, but for all corresponding hadronic interaction cross sections in the EAS above the chosen transition energy of $10^{15} \mathrm{eV}$.

\section{Secondary Multiplicity}

To change the multiplicity of secondaries, first of all, the leading particle needs to be excluded to prevent a change of the elasticity at the same time. The remaining particles are grouped together with respect to their type: nucleons, pions, kaons, photons, electrons and muons. To obtain a different multiplicity, particles are removed or duplicated at random from these groups depending on the aim of increasing $\left(f\left(E, f_{19}\right)>1\right)$ or decreasing
$\left(f\left(E, f_{19}\right)<1\right)$ the particle multiplicity. After the particle number has been adapted to be

$$
n_{\text {mult }}^{\text {mod }}=n_{\text {mult }}^{\text {orig }} f\left(E, f_{19}\right)
$$

the kinetic energy of all particles is scaled to yield exactly the same total energy as prior to the resampling. Since the leading particle is spared from all these operations, the elasticity of the interaction is conserved. Furthermore, this assures energy conservation as well as a constant energy ratio between the particle type groups. To conserve the charge, particles are changed to their anti-partners within particle type groups, until the total charge balance is restored up to a maximum charge offset of \pm 1 . Finally, all particle momenta are re-computed to account for their modified energies.

This minimalistic approach is optimized to conserve as many features of the original interaction model as possible. The developed method allows us to resample secondary particles of hadronic interactions in order to increase or decrease the multiplicity with as few assumptions as possible. No particle energy spectra or any other modelling is involved. Of course artificial fluctuations can be introduced since only existing particles are deleted or duplicated. But the qualitative shape of the particle energy spectra, the energy ratio between particle groups, the charge balance and the total energy as well as the leading particle are preserved as far as possible.

We studied the impact of the multiplicity modification on high statistics simulations of proton-air collisions at $10^{19} \mathrm{eV}$. In Fig. 15] (left) it can be seen that modifications of the multiplicity basically just scale the $\mathrm{d} N_{\mathrm{ch}} / \mathrm{d} \eta$ distribution. The fact that the leading particles are preserved can be clearly seen in Fig. 15 (middle) close to $X_{\text {lab }}=1$. To compensate this, there occurs an underrespectively over-shoot of the $\mathrm{d} N_{\mathrm{ch}} / \mathrm{d} X_{\text {lab }}$-distribution around $X_{\text {lab }} \sim 0.1$. At smaller $X_{\text {lab }}$ the scaling of the multiplicity can again be identified. Modifying the multiplicity has no impact on the resulting distribution of $N_{\text {neutral }} / N_{\text {charged }}$.

\section{Elasticity of Interactions}

To modify the elasticity $\kappa_{\mathrm{el}}=1-k_{\text {inel }}=E_{\text {leading }} / E_{\text {tot }}$ to obtain

$$
\kappa_{\mathrm{el}}^{\mathrm{mod}}=\kappa_{\mathrm{el}}^{\mathrm{orig}} f\left(E, f_{19}\right)
$$

it is only needed to re-distribute energy between the leading particle and the rest of the secondaries. Two facts are limiting the range of possible modifications of the elasticity:

lower bound: If the total available energy of all secondaries is distributed equally to all secondaries, then the minimal achievable elasticity, $\kappa_{\mathrm{el}}^{\bmod } \geq 1 / n_{\text {mult }}$, of this ensemble of particles is reached. This limit corresponds to a complete vanishing of any leading particle effects. All secondaries are emitted with an equal share of the total energy. 

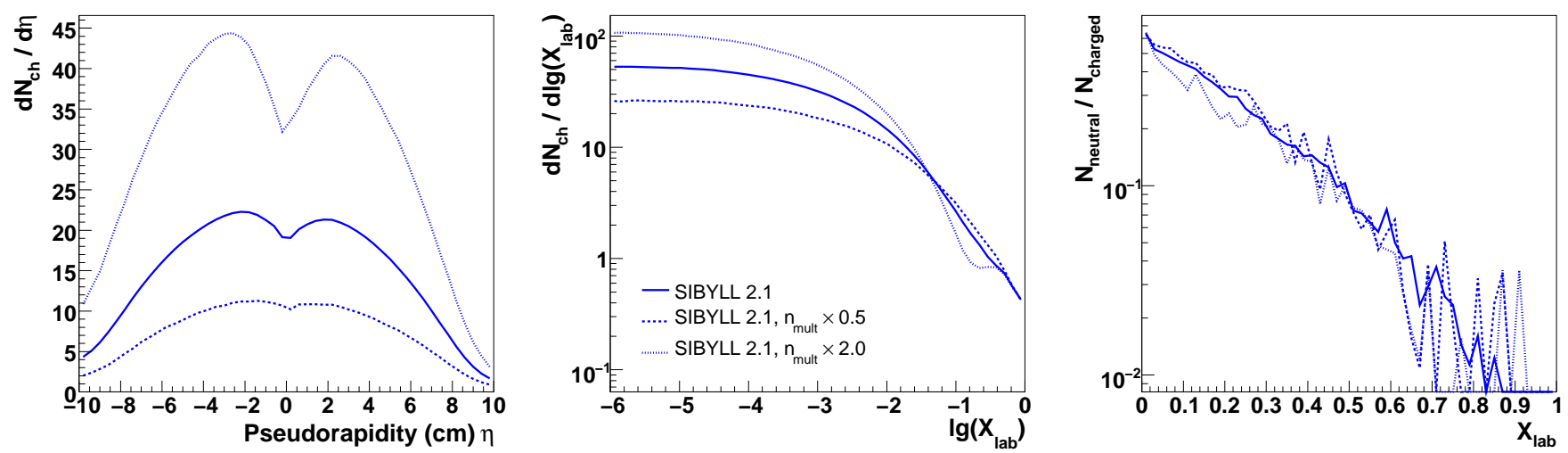

FIG. 15. Impact of modifying the multiplicity in proton-air collision at $10^{19} \mathrm{eV}$ on the resulting distribution of secondaries.
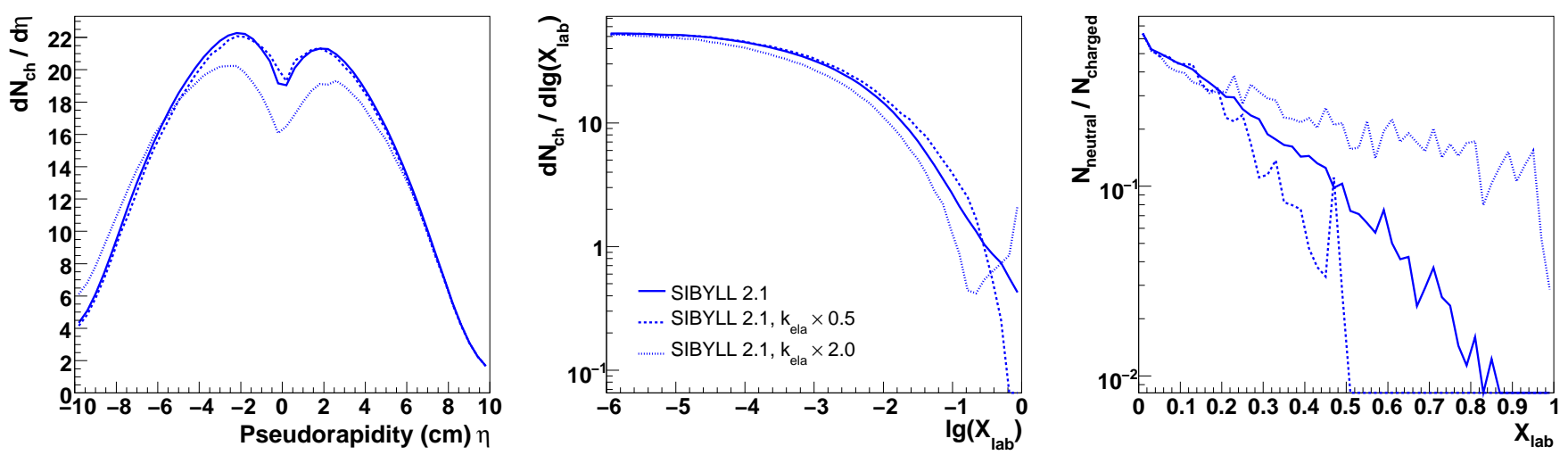

FIG. 16. Impact of modifying the elasticity in proton-air collision at $10^{19} \mathrm{eV}$ on the resulting distribution of secondaries.

upper bound: Since no secondary particles should get deleted the energy that is bound in the mass of all secondary particles is not accessible to further increase the elasticity of the leading particle. So the maximal elasticity is reached when the kinetic energy of all particles is transferred to the leading particle: $\kappa_{\mathrm{el}}^{\mathrm{mod}} \leq \sum_{i}^{\mathrm{n}_{\text {mult }}} E_{i}^{\mathrm{kin}} / E_{\mathrm{tot}}$. In the extreme case this corresponds to the production of particles with no kinetic energy. The leading particle takes the full energy of the projectile minus the energy that went into the mass of the other secondaries. Since these secondaries carry no kinetic energy they are thus not of any relevance for the development of the air shower cascade.

By design the total energy $E_{\text {tot }}$ is not changed during the procedure and since no particles are produced in addition to the existing ones or removed also the charge balance as well the particle type statistics are not altered. Because of the changing energy of all involved particles the particle momenta need to get re-computed at the end.

We studied the impact of modifying the elasticity on a high statistics simulation of proton-air collision at $10^{19} \mathrm{eV}$. In Fig. 16 (left+middle) it is shown that a smaller elasticity just removes the leading particles close to $X_{\text {lab }}=1$ and slightly increases the number of particles at smaller $X_{\text {lab }}$. In the central region of the collision there is basically no change. On the other hand, if the elasticity is increased, this leads to significantly reduced overall particle production, since more energy is carried away by the leading particles. This shows also clearly up in the central region of the collision. The seemingly large effect shown in Fig. 16 (right) is due in part to using a linear $X_{\text {lab }}$ axis compared to the logarithmic axis in Fig. 16 (middle).

\section{Charge-Ratio of Pions}

By far most of the hadronic particle production ends up in secondary pions. Since there are three types of pions, $\pi^{0}, \pi^{+}$and $\pi^{-}$, roughly a third of the produced particles are of each of the pion types. The charge ratio defined as

$$
c=\frac{n_{\pi^{0}}}{n_{\pi^{0}}+n_{\pi^{+}}+n_{\pi^{-}}},
$$

with $n_{\pi^{\times}}$being the number of pions of type $\pi^{\mathrm{x}}$, and is of the order of $1 / 3$. Since neutral pions decay al- 

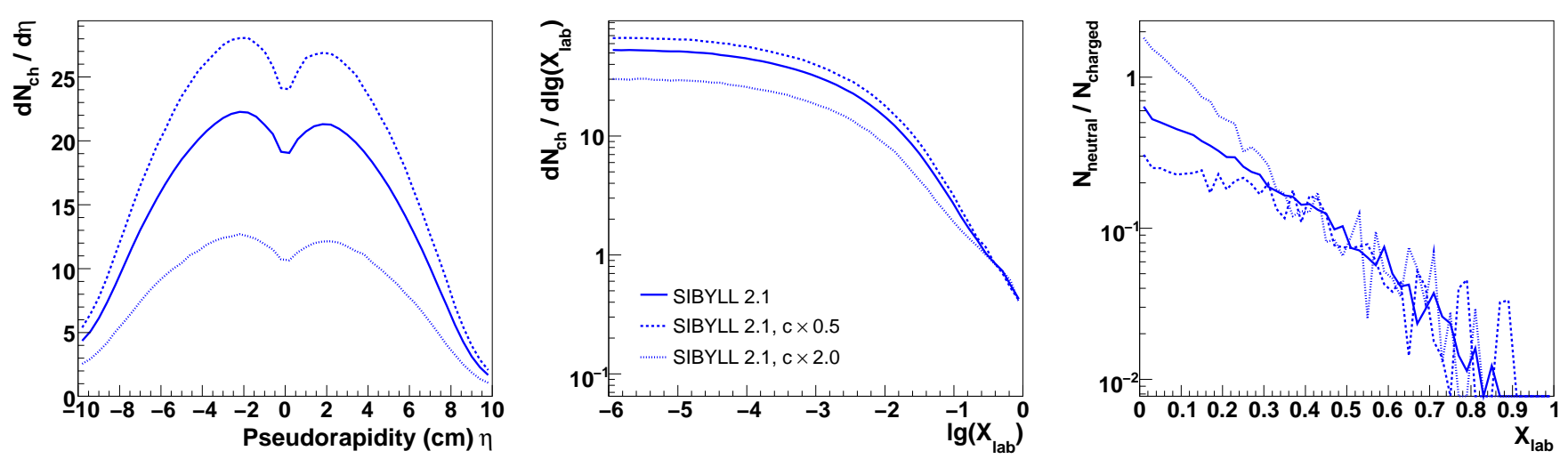

FIG. 17. Impact of modifying the charge ratio in proton-air collision at $10^{19} \mathrm{eV}$ on the resulting distribution of secondaries.

most instantly producing $2 \gamma$, this is the main channel to transfer energy into the electromagnetic cascade of the shower. The charged pions, on the other side, carry on the hadronic shower cascade (shower core) and eventually decay into muons.

We modify the charge ratio to finally yield

$$
c^{\text {mod }}=c^{\text {orig }} f\left(E, f_{19}\right) \text {, }
$$

by switching neutral into charged pions $\left(f\left(E, f_{19}\right)<1\right)$ or vice versa $\left(f\left(E, f_{19}\right)>1\right)$. We apply the following scheme:

positive projectiles: $\pi^{-} \leftrightarrow \pi^{0}$

negative projectiles: $\pi^{+} \leftrightarrow \pi^{0}$

neutral projectiles: $\pi^{ \pm} \leftrightarrow \pi^{0}$.

Furthermore, in all cases the leading particle is excluded and thus fully preserved.

Due to this logic there are limitations in the modification of the charge ratio of the form

$$
0<c^{\bmod }<\frac{n_{\pi^{0}}+n_{\pi^{\mathrm{src}}}}{n_{\pi^{0}}+n_{\pi^{-}}+n_{\pi^{+}}},
$$

where $n_{\pi^{\mathrm{src}}}$ is, depending on the projectile, $n_{\pi^{+}}$for negative, $n_{\pi^{-}}$for positive, and $n_{\pi^{+}}+n_{\pi^{-}}$for neutral projectiles. Given this scheme we find the probability for

$$
\begin{aligned}
& f\left(E, f_{19}\right)>1: \text { to switch one } \pi^{0} \rightarrow \pi^{\mathrm{src}} \text { is: } \\
& \quad p=\left(n_{\pi^{0}}-f\left(E, f_{19}\right) n_{\pi^{0}}\right) / n_{\pi^{0}}=1-f\left(E, f_{19}\right)
\end{aligned}
$$

and

$$
\begin{gathered}
f\left(E, f_{19}\right)<1: \text { to switch one } \pi^{\mathrm{src}} \rightarrow \pi^{0} \text { is: } \\
p=\left(f\left(E, f_{19}\right) n_{\pi^{0}}-n_{\pi^{0}}\right) / n_{\pi^{\mathrm{src}}} .
\end{gathered}
$$

Since changing the pion type of individual particles also slightly changes their rest mass, all momenta need to be recomputated given the new rest mass.

We studied the impact of modifying the charge ratio on a high statistics simulation of proton-air collision at $10^{19} \mathrm{eV}$. In Fig. 17 (left) it is shown that the charge ratio just scales the $\mathrm{d} N_{\mathrm{ch}} / \mathrm{d} \eta$-distribution, while in Fig. 17 (middle) it is also clear that our strategy to preserve the leading particle effect works, since there is no impact on $\mathrm{d} N_{\mathrm{ch}} / \mathrm{d} X_{\text {lab }}$ close to $X_{\text {lab }}=1$. Similarly Fig. 17 (right) illustrates how the leading particles at high $X_{\text {lab }}$ are preserved and the charge ratio only changes at small $X_{\text {lab }}$.

\section{Appendix B: Nucleus Projectiles}

For projectiles that are nuclei of $A$ nucleons the modification of interaction characteristics are performed within the Glauber theory [44, 45]. With the semi-superposition model [78] it is calculated how many of the nucleons of

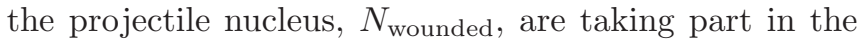
interaction with the target nucleus (air). The interaction of these nucleons with the target are then computed individually, and can thus be rescaled by the same techniques as introduced in Appendix $\mathrm{A}$, at the reduced projectile energy of $E_{0} / A$.

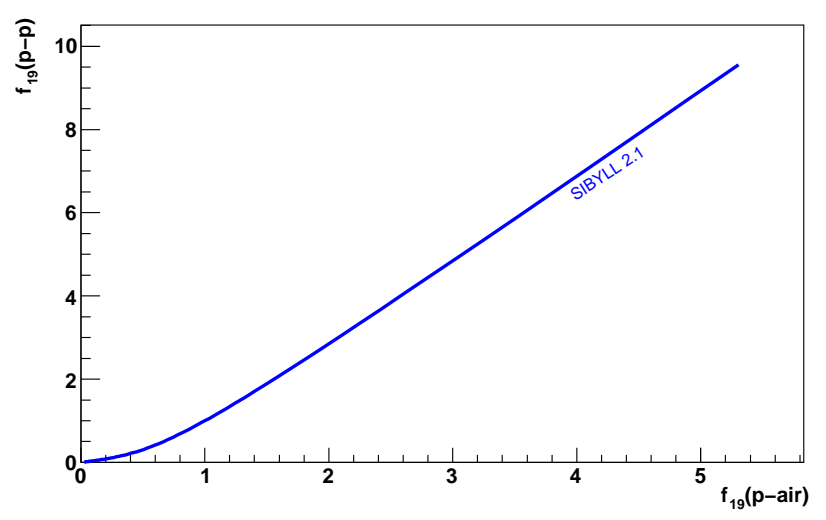

FIG. 18. Conversion from $f_{19}$ to $f_{19}^{\mathrm{p}-\mathrm{p}}$ using the Glauber theory within SiBYLL. 
The cross sections for nucleus primaries are also computed within the Glauber framework. The nucleusnucleus cross section is computed based on the fundamental parameters $\sigma_{\text {tot }}^{\mathrm{p}-\mathrm{p}}, \sigma_{\mathrm{el}}^{\mathrm{p}-\mathrm{p}}$ and $B_{\mathrm{el}}$, which are scaled for our purpose to

$$
\begin{aligned}
\sigma_{\text {tot }, \bmod }^{\mathrm{p}-\mathrm{p}} & =f\left(E, f_{19}^{\mathrm{p}-\mathrm{p}}\right) \sigma_{\text {tot }}^{\mathrm{p}-\mathrm{p}} \\
\sigma_{\text {ela }, \bmod }^{\mathrm{p}-\mathrm{p}} & =f\left(E, f_{19}^{\mathrm{p}-\mathrm{p}}\right) \sigma_{\text {el }}^{\mathrm{p}-\mathrm{p}} \\
B_{\text {ela }, \bmod } & =f\left(E, f_{19}^{\mathrm{p}-\mathrm{p}}\right) B_{\text {el }}
\end{aligned}
$$

where $f_{19}^{\mathrm{p}-\mathrm{p}}$ is chosen to yield the following equation

$$
f_{19}=\frac{\sigma_{\text {prod }}^{\mathrm{p}-\text { air }}\left(\sigma_{\text {tot }, \text { mod }}^{\mathrm{p}-\mathrm{p}}, \sigma_{\mathrm{ela}, \bmod }^{\mathrm{p}-\mathrm{p}}, B_{\mathrm{ela}, \bmod }\right)}{\sigma_{\text {prod }}^{\mathrm{p}-\text { air }}\left(\sigma_{\mathrm{tot}}^{\mathrm{p}-\mathrm{p}}, \sigma_{\mathrm{el}}^{\mathrm{p}-\mathrm{p}}, B_{\mathrm{el}}\right)} .
$$

The proton-air cross sections are all computed in the Glauber framework as implemented in SiBYLL. This approach assures consistency with the rest of the hadronic cross sections in the air shower that are modified according to Eq. A1 . The relation between $f_{19}$ and the rescaling parameter of the fundamental proton-proton interaction parameters $f_{19}^{\mathrm{p}-\mathrm{p}}$ is shown in Fig. 18
[1] J. Abraham et al. (Pierre Auger), Nucl. Instrum. Meth. A523, 50 (2004).

[2] H. Kawai et al. (TA), Nucl. Phys. Proc. Suppl. 175-176, 221 (2008).

[3] R. Abbasi et al. (HiRes), Astrophys. J. 622, 910 (2005), astro-ph/0407622.

[4] B. R. Dawson, R. Meyhandan, and K. M. Simpson, Astropart. Phys. 9, 331 (1998), astro-ph/9801260.

[5] M. Ave et al., Astropart. Phys. 19, 61 (2003), astro-ph/0203150

[6] M. T. Dova, M. E. Mancenido, A. G. Mariazzi, T. P. McCauley, and A. A. Watson, Astropart. Phys. 21, 597 (2004), arXiv:astro-ph/0312463

[7] J. Abraham et al. (Pierre Auger), Proc. of $31^{\text {th }}$ Int. Cosmic Ray Conf., Lodz, Poland(2009), arXiv:0906.2319 [astro-ph]

[8] J. Abraham et al. (Pierre Auger), Phys. Rev. Lett. 104, 091101 (2010), arXiv:1002.0699 [astro-ph].

[9] T. Antoni et al. (KASCADE), Astropart. Phys. 24, 1 (2005), astro-ph/0505413

[10] M. Nagano et al., J. Phys. G18, 423 (1992).

[11] M. Takeda et al., Phys. Rev. Lett. 81, 1163 (1998), astro-ph/9807193.

[12] M. Ave, J. Knapp, J. Lloyd-Evans, M. Marchesini, and A. A. Watson, Astropart. Phys. 19, 47 (2003), astro-ph/0112253

[13] M. Aglietta et al. (EAS-TOP), Astropart. Phys. 21, 583 (2004).

[14] M. Amenomori et al. (Tibet AS $\gamma$ ), Astrophys. J. 678, 1165 (2008), arXiv:0801.1803 [hep-ex]

[15] D. Heck, "The influence of hadronic interaction models on simulated air-showers: A phenomenologic comparison," (2008), talk given at CORSIKA School 2008, Freudenstadt, Germany, 25 - 30 November 2008, available at http://www-ik.fzk.de/corsika/corsikaschool2008/.

[16] B. Rossi and K. Greisen, Rev. Mod. Phys. 13, 240 (1941).

[17] M. Giller, A. Kacperczyk, J. Malinowski, W. Tkaczyk, and G. Wieczorek, J. Phys. G31, 947 (2005).

[18] F. Nerling, J. Blümer, R. Engel, and M. Risse, Astropart. Phys. 24, 421 (2006).

[19] F. Schmidt, M. Ave, L. Cazon, and A. S.
Chou, Astropart. Phys. 29, $355 \quad$ (2008), arXiv:0712.3750 [astro-ph].

[20] P. Lipari, Phys. Rev. 79, 063001 (2008), arXiv:0809.0190 [astro-ph]

[21] S. Lafebre et al., Astropart. Phys. 31, 243 (2009), arXiv:0902.0548 [astro-ph.HE]

[22] J. Matthews, Astropart. Phys. 22, 387 (2005).

[23] R. Engel, Nucl. Phys. Proc. Suppl. 82, 221 (2000).

[24] A. Donnachie and P. Landshoff, Phys. Lett. B296, 227 (1992), hep-ph/9209205

[25] G. Pancheri, R. M. Godbole, A. Grau, and Y. N. Srivastava, Acta Phys. Polon. B38, 2979 (2007), arXiv:hep-ph/0703174

[26] P. Landshoff, Lectures at School on QCD, Calabria(July 2007), arXiv:0709.0395 [hep-ph]

[27] P. V. Landshoff(2009), arXiv:0903.1523 [hep-ph].

[28] R. Ulrich, R. Engel, S. Muller, F. Schussler, and M. Unger, Nucl. Phys. Proc. Suppl. 196, 335 (2009), arXiv:0906.3075 [astro-ph]

[29] S. Ostapchenko, Nucl. Phys. Proc. Suppl. 151, 143 (2006), hep-ph/0412332

[30] S. Ostapchenko, Phys. Lett. B636, 40 (2006), hep-ph/0602139

[31] K. Werner and T. Pierog, AIP Conf. Proc. 928, 111 (2007), arXiv:0707.3330 [astro-ph].

[32] N. Kalmykov and S. Ostapchenko, Phys. Atom. Nucl. 56, 346 (1993).

[33] N. Kalmykov and S. Ostapchenko, Sov. J. Nucl. Phys. 50, 315 (1989).

[34] E.-J. Ahn, R. Engel, T. K. Gaisser, P. Lipari, and T. Stanev, Phys. Rev. D80, 094003 (2009), arXiv:0906.4113 [hep-ph],

[35] T. Bergmann et al., Astropart. Phys. 26, 420 (2007), astro-ph/0606564

[36] H. Drescher, M. Hladik, S. Ostapchenko, T. Pierog, and K. Werner, Phys. Rept. 350, 93 (2001), hep-ph/0007198.

[37] M. Hladik, H. Drescher, S. Ostapchenko, T. Pierog, and K. Werner, Phys. Rev. Lett. 86, 3506 (2001), hep-ph/0102194

[38] J. N. Capdevielle, J. Phys. G15, 909 (1989).

[39] J. Ranft, Phys. Rev. D51, 64 (1995).

[40] K. Werner, Phys. Rep. 232, 87 (1993).

[41] D. Heck et al., Proc. of 27th Int. Cosmic Ray Conf., Ham- 
burg 1, 233 (2001).

[42] T. Antoni et al. (KASCADE), Astropart. Phys. 16, 245 (2002), astro-ph/0102443

[43] T. Abu-Zayyad et al. (HiRes-MIA), Phys. Rev. Lett. 84, 4276 (2000), astro-ph/9911144.

[44] R. Glauber, Phys. Rev. 100, 242 (1955).

[45] R. Glauber and G. Matthiae, Nucl. Phys. B21, 135 (1970).

[46] T. Pierog and K. Werner, Proc. of $30^{\text {th }}$ Int. Cosmic Ray Conf., Merida, Mexico(2007), astro-ph/0611311.

[47] T. Pierog and K. Werner, Proc. of 30th Int. Cosmic Ray Conf., Merida 4, 629 (2007).

[48] K. Aamodt et al. (ALICE), Phys. Rev. Lett. 105, 072002 (2010), arXiv:1006.5432 [hep-ex].

[49] K. Aamodt et al. (ALICE), Eur. Phys. J. C68, 89 (2010), arXiv:1004.3034 [hep-ex],

[50] K. Aamodt et al. (ALICE), Eur. Phys. J. C68, 345 (2010), arXiv:1004.3514 [hep-ex].

[51] K. Aamodt et al. (ALICE)(2009), arXiv:0911.5430 [hep-ex].

[52] G. Aad et al. (ATLAS), Phys. Lett. B688, 21 (2010), arXiv:1003.3124 [hep-ex],

[53] V. Khachatryan et al. (CMS), JHEP 02, 041 (2010), arXiv:1002.0621 [hep-ex].

[54] V. Khachatryan et al. (CMS), Phys. Rev. Lett. 105, 022002 (2010), arXiv:1005.3299 [hep-ex]

[55] V. Khachatryan et al. (CMS)(2010), arXiv:1006.2083 [hep-ex],

[56] D. d'Enterria, R. Engel, S. Ostapchenko, T. Pierog, and K. Werner(2010), in preparation.

[57] For a historical account of the development of this model see 89].

[58] W. Heitler, Rev. Mod. Phys. 21, 113 (1949).

[59] T. Pierog, R. Engel, and D. Heck, Czech. J. Phys. 56, A161 (2006), astro-ph/0602190.

[60] T. K. Gaisser, Cosmic rays and particle physics (Cambridge Univ. Press, Cambridge, 1990).

[61] J. Alvarez-Muniz, R. Engel, T. K. Gaisser, J. A. Ortiz, and T. Stanev, Phys. Rev. D66, 033011 (2002), astro-ph/0205302

[62] C. Meurer, J. Blümer, R. Engel, A. Haungs, and M. Roth, Czech. J. Phys. 56, A211 (2006), astro-ph/0512536

[63] It is a feature of air shower universality (see, for example, [16 21]) that $\Lambda$ does not depend on the primary particle or hadronic interaction model.

[64] J. Alvarez-Muniz, R. Engel, T. Gaisser, J. Ortiz, and T. Stanev, Phys. Rev. D66, 033011 (2002), astro-ph/0205302.

[65] J. Bellandi, R. J. M. Covolan, and A. L. Godoi, Phys. Lett. B343, 410 (1995).
[66] R. Engel, Nucl. Phys. Proc. Suppl. 122, 40 (2003).

[67] S. S. Ostapchenko, J. Phys. G29, 831 (2003).

[68] S. Ostapchenko, Czech. J. Phys. 56, A149 (2006), arXiv:hep-ph/0601230

[69] C. Bleve, R. Parsons, J. Knapp, and S. Ostapchenko, Proc. of $31^{\text {th }}$ Int. Cosmic Ray Conf., Lodz, Poland(2009).

[70] J. Knapp, D. Heck, and G. Schatz, "Comparison of hadronic interaction models used in air shower simulations and of their influence on shower development and obsevables," (1996), in Wissenschaftliche Berichte FZKA 5828, Forschungszentrum Karlsruhe.

[71] J. Knapp, D. Heck, S. Sciutto, M. Dova, and M. Risse, Astropart. Phys. 19, 77 (2003), astro-ph/0206414

[72] L. A. Anchordoqui, M. T. Dova, L. N. Epele, and S. J. Sciutto, Phys. Rev. D59, 094003 (1999), arXiv:hep-ph/9810384

[73] C. A. Garcia Canal, S. J. Sciutto, and T. Tarutina, Phys. Rev. D79, 054006 (2009), arXiv:0903.2409 [astro-ph.HE]

[74] J. Blümer et al. (Pierre Auger), New J. Phys. 12, 035001 (2010).

[75] A. Santangelo and A. Petrolini, New J. Phys. 11, 065010 (2009).

[76] R. Abbasi et al. (HiRes), Phys. Rev. Lett. 100, 101101 (2008), astro-ph/0703099

[77] J. Abraham et al. (Pierre Auger), Phys. Rev. Lett. 101, 061101 (2008), arXiv:0806.4302 [astro-ph].

[78] J. Engel, T. Gaisser, T. Stanev, and P. Lipari, Phys. Rev. D46, 5013 (1992).

[79] R. Fletcher, T. Gaisser, P. Lipari, and T. Stanev, Phys. Rev. D50, 5710 (1994).

[80] Available upon request from ralf.ulrich@kit.edu.

[81] H.-J. Drescher, Phys. Rev. D77, 056003 (2007), arXiv:0712.1517 [hep-ph],

[82] H.-J. Drescher, M. Bleicher, S. Soff, and H. Stoecker, Astropart. Phys. 21, 87 (2004), astro-ph/0307453.

[83] I. C. Maris et al., Nucl. Phys. Proc. Suppl. 196, 86 (2009), arXiv:0907.0409 [astro-ph.CO].

[84] C. Song et al. (HiRes), Astropart. Phys. 14, 7 (2000), astro-ph/9910195

[85] H. M. J. Barbosa, F. Catalani, J. A. Chinellato, and C. Dobrigkeit, Astropart. Phys. 22, 159 (2004), arXiv:astro-ph/0310234

[86] D. Harari, S. Mollerach, and E. Roulet, JCAP 0611, 012 (2006), arXiv:astro-ph/0609294.

[87] D. Allard, N. G. Busca, G. Decerprit, A. V. Olinto, and E. Parizot, JCAP 0810, 033 (2008), arXiv:0805.4779 [astro-ph]

[88] www.campusgrid.de.

[89] J. Linsley, Sci. Am. 239, 48 (1978). 